\title{
Effects of chiral effective field theory equation of state on binary neutron star mergers
}

\author{
Andrea Endrizzi, ${ }^{1,2}$ Domenico Logoteta, ${ }^{3,4}$ Bruno Giacomazzo, ${ }^{1,2}$ Ignazio Bombaci, ${ }^{3,4}$ \\ Wolfgang Kastaun, ${ }^{5,6}$ and Riccardo Ciolfi ${ }^{7,2}$ \\ ${ }^{1}$ Physics Department, University of Trento, via Sommarive 14, I-38123 Trento, Italy \\ ${ }^{2}$ INFN-TIFPA, Trento Institute for Fundamental Physics and Applications, \\ via Sommarive 14, I-38123 Trento, Italy \\ ${ }^{3}$ Dipartimento di Fisica "E. Fermi", Università di Pisa, Largo B. Pontecorvo 3, I-56127 Pisa, Italy \\ ${ }^{4}$ INFN, Sezione di Pisa, Largo B. Pontecorvo 3, I-56127 Pisa, Italy \\ ${ }^{5}$ Max Planck Institute for Gravitational Physics (Albert Einstein Institute), \\ Callinstrasse 38, 30167 Hannover, Germany \\ ${ }^{6}$ Leibniz Universität Hannover, Institute for Gravitational Physics, \\ Callinstrasse 38, 30167 Hannover, Germany \\ ${ }^{7}$ INAF, Osservatorio Astronomico di Padova, Vicolo dell'Osservatorio 5, I-35122 Padova, Italy
}

(Received 25 June 2018; published 16 August 2018)

\begin{abstract}
We present fully general relativistic simulations of binary neutron star mergers, employing a new zerotemperature chiral effective field theory equation of state (EOS), the BL EOS. We offer a comparison with respect to the older GM3 EOS, which is based on standard relativistic mean-field theory, and separately determine the impact of the mass. We provide a detailed analysis of the dynamics, with focus on the postmerger phase. For all models, we extract the gravitational wave strain and the postmerger frequency spectrum. Further, we determine the amount, velocity, and polar distribution of ejected matter and provide estimates for the resulting kilonova signals. We also study the evolution of the disk while it is interacting with the hypermassive remnant and discuss the merits of different disk mass definitions applicable before collapse, with regard to the mass remaining after black hole formation. Finally, we investigate the radial mass distribution and rotation profile of the remnants, which validate previous results and also corroborate a recently proposed stability criterion.
\end{abstract}

DOI: 10.1103/PhysRevD.98.043015

\section{INTRODUCTION}

The first detections of gravitational waves (GWs) by the advanced LIGO network have officially started the era of GW astronomy [1]. On August 1, 2017, advanced Virgo also joined the search for GW sources and on August 17, 2017, the first GW signal from a binary neutron star (BNS) coalescence was detected [2]. The GW detection was followed by a large number of electromagnetic counterparts observed by ground- and space-based telescopes [3,4]. Those confirm that (at least some) short gamma-ray bursts (SGRBs) are indeed associated with binary neutron star mergers [5] and provide evidence that such systems are also the source of the heaviest elements in the Universe $[3,6]$. Unfortunately, the current sensitivity of the Virgo and LIGO detectors was not sufficient to detect a postmerger GW signal [7] and, therefore, it is not clear if a black hole $(\mathrm{BH})$ or a neutron star (NS) were the result of the merger. There is, however, ongoing analysis on the subject combining the GW emission with its EM counterparts. For example, kilonova models and observations (e.g., see [8-10]) seem to exclude a long-lived NS remnant and support the formation of a short-lived hypermassive NS after merger. Moreover, if an NS, either hypermassive or supramassive, was formed after the merger, an observation of its GW emission could have been used to put strong constraints on the equation of state (EOS) of NS matter [11]. Nevertheless, preliminary constraints for the EOS were derived from tidal effects during the inspiral [2]. Note, however, that these constraints strongly rely on the accuracy of the waveform models. Further detectors, LIGO India and KAGRA, will be added to the GW detector network in the next years. Third generation detectors with higher sensitivities, such as the Einstein Telescope, are planned for the future. Such detectors may increase the probability to detect postmerger GW emission as well as explore regimes which cannot be studied during the inspiral (e.g., study EOS at larger densities and temperatures [12]).

BNS systems can be classified based on their total baryonic mass and EOS. BNS systems with a total mass higher than the maximum mass that can be supported by uniform rotation (see [13]) could promptly form a BH after merger (if the mass is sufficiently high [14]) or form an 
hypermassive neutron star (HMNS) which can survive for up to $\sim 1 \mathrm{~s}$ before collapsing to a BH. BNS systems with a total mass below the maximum mass for uniformly rotating NSs, but above the maximum mass for nonrotating NSs, will instead produce a supramassive neutron star (SMNS). A SMNS collapses once gravitational waves and magnetic fields have decreased the angular momentum below a critical limit (which depends on mass and EOS). A typical SMNS can therefore survive for hours or longer. If the total mass of the BNS system is even below the maximum mass of a nonrotating NS, the merger remnant is a stable NS [15].

Two observations of $\sim 2 \mathrm{M}_{\odot}$ NSs seem to indicate that a significant fraction of BNS mergers could lead to the formation of HMNS or SMNS after merger, depending on the NS EOS, since the most common NS masses measured in galactic BNS systems are $\sim 1.35 \mathrm{M}_{\odot}[16]$.

In this work we performed general relativistic hydrodynamic simulations of equal mass BNS systems with different EOSs. For each, we chose initial values for the NS masses which slightly exceed the maximum mass for a uniformly rotating NS. The motivation is to study the HMNS case with different EOSs.

In the present paper we employed a new EOS for nuclear matter in $\beta$-equilibrium at zero temperature (BL EOS in the following) described in [17]. It uses the Brueckner-BetheGoldstone (BBG) [18-20] many-body theory with realistic two-body and three-body nuclear interactions derived in the framework of chiral effective field theory (ChEFT). This new EOS is used here for the first time to carry out general relativistic hydrodynamic simulations of merging BNSs. For comparison, we also used a standard relativistic mean-field EOS model for nuclear matter in $\beta$-equilibrium, specifically the GM3 parametrization of the Glendenning-Moszkowski $[21,22]$ EOS. Note that in comparison to the BL EOS, the GM3 EOS leads to NS with lower compactness and higher tidal deformability. Although the latter approaches preliminary constraints obtained from GW170817 [2], we deemed this choice useful for studying EOS-related differences.

The paper is organized as follows. In Sec. II, we give a more in-depth description of the new ChEFT EOS and explore the main differences to the standard relativistic mean-field one. In Sec. III, we describe the initial data and the numerical setup used for the simulations. In Sec. IV, we analyze the general dynamics of the BNS systems during inspiral and postmerger, and the rotation profile of the remnants. In Sec. V, we discuss the EOS impact on the mass ejection and the consequences for the corresponding kilonova signal. In Sec. VI, we present the GW signals and discuss the main differences introduced by the new EOS. In Sec. VII, we present our main conclusions.

Unless specified otherwise we use a system of units in which $G=c=M_{\odot}=1$.

\section{THE BL EQUATION OF STATE}

In the present work, we model the core of the two merging neutron stars as a uniform charge-neutral fluid of neutrons $(n)$, protons $(p)$, electrons $\left(e^{-}\right)$and muons $\left(\mu^{-}\right)$in equilibrium with respect to the weak interaction $(\beta$-stable nuclear matter). We neither consider the possible existence of "exotic" constituents in the stellar core, such as hyperons $[23,24]$, nor the possibility of a deconfined quark phase $[22,25,26]$.

The BL EOS has been very recently derived in [17], making use of the Brueckner-Bethe-Goldstone (BBG) quantum many-body theory in the Brueckner-HartreeFock (BHF) approximation (see e.g., [27,28] and references quoted therein). The main innovative aspect of this EOS is the use of two-body and three-body nuclear interactions derived within the framework of the so-called chiral effective field theory (ChEFT). This is a different approach with respect to other well-known microscopic EOS models for $\beta$-stable nuclear matter, as e.g., the APR [29] EOS or the BBB [30] EOS. In fact, ChEFT has opened a new route for the description of nuclear interactions [31-37] consistent with quantum chromodynamics (QCD), the fundamental theory of the strong interaction. The most notable advantage of this method is that two-body, threebody, and even many-body nuclear interactions can be calculated perturbatively, i.e., order by order, according to a well-defined scheme. The latter is based on a low-energy effective QCD Lagrangian which preserves the symmetries of QCD and particularly the approximate chiral symmetry. Within this chiral perturbation theory (ChPT) the details of the QCD dynamics are contained in parameters which are fixed by low-energy experimental data. This systematic technique is especially advantageous in the case of nuclear systems where the relevance of the three-nucleon force (TNF) is a well-established feature. In fact, it is well known that TNFs are essential to reproduce the experimental binding energy of few-nucleon $(\mathrm{A}=3,4)$ systems [38-40] and the empirical saturation point $\left(n_{0}=0.16 \pm 0.01 \mathrm{fm}^{-3}\right.$, $E /\left.A\right|_{n_{0}}=-16.0 \pm 1.0 \mathrm{MeV}$ ) of symmetric nuclear matter (SNM) $[29,30,41,42]$. In addition, TNFs are crucial in the case of dense $\beta$-stable nuclear matter to obtain a sufficiently stiff EOS $[29,30,43]$ compatible with the measured masses, $M=1.97 \pm 0.04 M_{\odot}$ [44] and $M=2.01 \pm 0.04 M_{\odot}$ [45] of the neutron stars in PSR J1614 - 2230 and PSR J0348 + 0432, respectively.

In addition to the BL EOS, for the purpose of comparison, we also used a standard relativistic mean-field EOS (GM3 [21,22]). To model the NS crust we used the EOS reported in Ref. [46] for the outer crust and the EOS reported in Ref. [47] for the inner crust.

The calculated values of the saturation points of SNM for the BL and GM3 EOS models are reported in Table I. Both models give saturation points in very good agreement with the empirical ones. In addition, the calculated gravitational maximum mass $M_{g}^{\mathrm{TOV}}$ for nonrotating NS (see Table I) for the two EOS models is almost the same (relative difference $\sim 3 \%$ ) indicating that the overall stiffness of the two EOS is very similar. Obviously this result does not imply that the 
TABLE I. Properties of nuclear matter for the EOS models used in this work: saturation density $n_{0}$ and corresponding energy per nucleon $E / A$ for symmetric nuclear matter, as well as symmetry energy $E_{\text {sym }}^{0}$ and its slope parameter $L$ at the calculated saturation density. Further, $M_{g}^{\mathrm{TOV}}$ is the maximum gravitational mass of a nonrotating NS. $M_{g}^{\text {SMNS }}$ and $M_{b}^{\text {SMNS }}$ are the maximum gravitational and baryonic masses, respectively, for a uniformly rotating NS.

\begin{tabular}{lccccccc}
\hline \hline Model & $n_{0}\left(\mathrm{fm}^{-3}\right)$ & $E / A(\mathrm{MeV})$ & $E_{\mathrm{sym}}^{0}(\mathrm{MeV})$ & $L(\mathrm{MeV})$ & $M_{g}^{\mathrm{TOV}}\left[M_{\odot}\right]$ & $M_{g}^{\mathrm{SMNS}}\left[M_{\odot}\right]$ & $M_{b}^{\mathrm{SMNS}}\left[M_{\odot}\right]$ \\
\hline BL & 0.171 & -15.23 & 35.39 & 76.0 & 2.08 & 2.44 & 2.84 \\
GM3 & 0.153 & -16.32 & 32.40 & 89.7 & 2.01 & 2.36 & 2.70 \\
\hline \hline
\end{tabular}

two EOS models will give similar results for other neutron star properties (e.g., mass-radius relation, moment of inertia, mass shed frequency, tidal deformability, etc) or in binary neutron star mergers simulations.

The nuclear symmetry energy $E_{\text {sym }}[48,49]$, and particularly its density dependence, is another important physical quantity which influences the properties of asymmetric nuclear matter (i.e., matter with $n_{n} \neq n_{p}$, where $n_{n}$ and $n_{p}$ denote the neutron and proton number densities, respectively). In particular, the symmetry energy determines the proton fraction and the pressure of nuclear matter in $\beta$-equilibrium [42,50]. Consequently, it has an impact on many NS attributes such as the radius, the moment of inertia, and the tidal deformability [51,52].

The symmetry energy can be obtained [50] taking the difference between the energy per nucleon $E / A$ of pure neutron matter and the one of SNM at a given total nucleon number density $n=n_{n}+n_{p}$. The symmetry properties of nuclear matter around the saturation density $n_{0}$ are summarized by the value of $E_{\text {sym }}^{0} \equiv E_{\text {sym }}\left(n_{0}\right)$ and by the value of the so-called symmetry energy slope parameter:

$$
L=\left.3 n_{0} \frac{\partial E_{\mathrm{sym}}(n)}{\partial n}\right|_{n_{0}}
$$

It has been shown [53] that a strong correlation between the values of $E_{\text {sym }}^{0}$ and $L$ can be deduced in a nearly modelindependent way from nuclear binding energies. In addition, it has been recently demonstrated [54] that the unitary gas limit [55], which can be used to describe low density neutron matter, puts stringent constraints on the possible values of the symmetry energy parameters, excluding a large region in the $E_{\mathrm{sym}}^{0}-L$ plane (see Fig. 3 in Ref. [54]). As pointed out by the authors of Ref. [54]) several tabulated EOS currently used in astrophysical simulations of supernova explosions and BNS mergers violate the unitary gas bounds. Thus the unitary gas model can be used as a novel way to constrain dense matter EOS to be used in astrophysical applications.

The values of $E_{\mathrm{sym}}^{0}$ and $L$ calculated for the BL and GM3 EOS models are reported in Table I. These values are compatible with the unitary gas bound given in Ref. [54] for the case of the BLEOS whereas they are not compatible with the unitary gas bound in the case of the GM3 EOS.
The differences between the two EOSs in terms of mass versus rest mass density relation are shown in Fig. 1. As one can see, the ChEFT EOS can support a higher maximum mass than the GM3 EOS. Both EOSs allow NSs with gravitational mass above $2 M_{\odot}$, compatible with current observational lower limits on the maximum mass [44,45]. In Fig. 2, we show the gravitational mass versus radius for maximally uniformly rotating and nonrotating NSs described by the EOSs used in this paper. The BL EOS leads to more compact stars than the GM3 EOS: for a NS with $M_{g}=1.35 M_{\odot}$, the radius is around $1 \mathrm{~km}$ smaller.

The BL and GM3 EOS do not include thermal effects. For the evolution, we add a thermal component using a standard gamma-law prescription, described in Appendix. Such hybrid EOSs are often used in the field of BNS simulations, see [56,57].

Note that the EOS assume $\beta$-equilibrium, and do not provide composition dependency out of $\beta$-equilibrium. However, the matter will not stay in $\beta$-equilibrium during the rapid evolution at merger. While the density changes rapidly, the electron fraction changes on timescales given by weak processes. The presence of trapped neutrinos in high density regions, and particularly their influence on the matter composition (particle fractions) and their contribution to the pressure further complicates the picture [58,59].

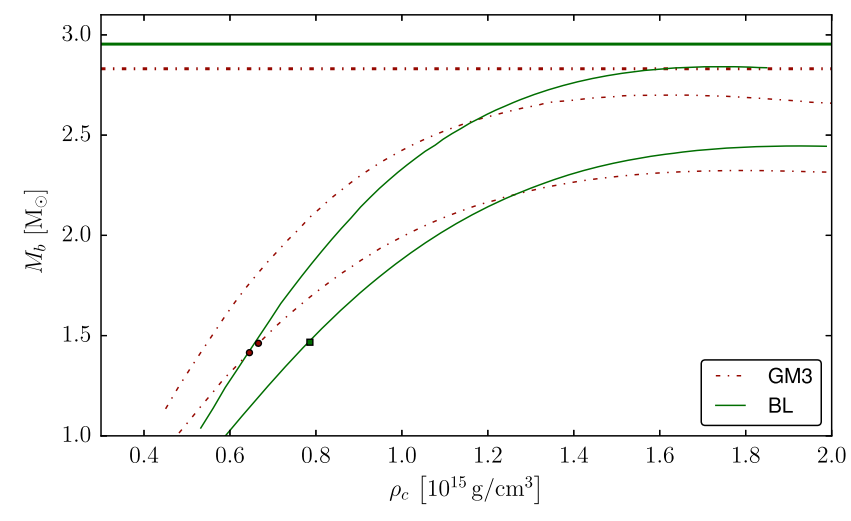

FIG. 1. Baryonic mass as a function of central rest mass density of nonrotating (lower curves) and maximally uniformly rotating (upper curves) NSs for the two EOSs used in this paper (BL and GM3). The horizontal lines represent the masses of the BNS systems, while the markers on the curves represent the mass values for the single NSs in the binary. 

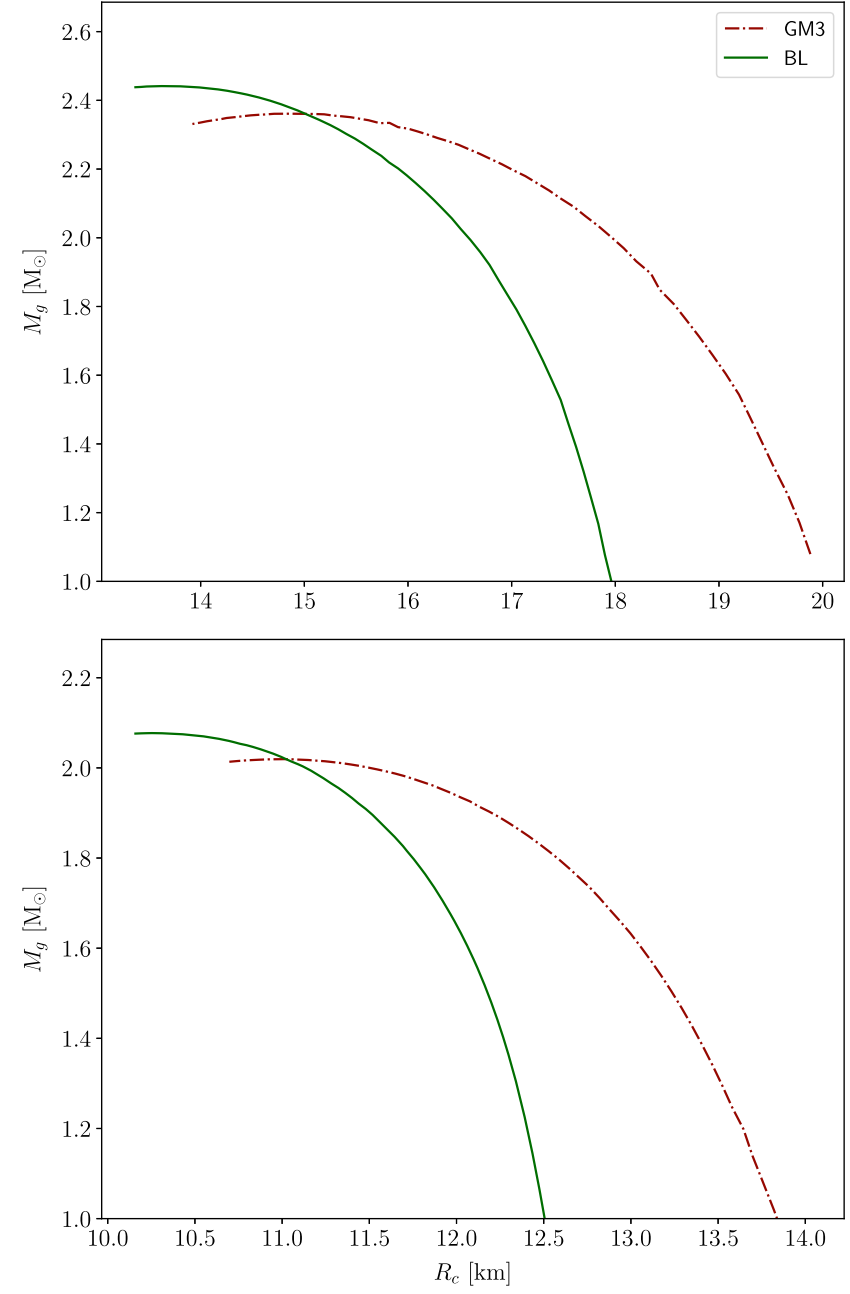

FIG. 2. Gravitational mass as a function of circumferential radius of NSs depending on the chosen EOS. Bottom panel: relation for nonrotating NSs. Top panel: relation for NSs rotating uniformly at mass shedding limit.

By using the BL or GM3 EOS, we will make the implicit approximation that $\beta$-equilibrium holds during merger.

\section{INITIAL DATA AND NUMERICAL SETUP}

In this work we evolve an unmagnetized equal-mass BNS system (with gravitational masses $1.35 M_{\odot}$ ) employing the new BL EOS. We also study two BNS systems with the older GM3 EOS. One has the same gravitational masses as the BL model. The other is $3 \%$ lighter, such that the ratio $M_{\mathrm{b}}^{\text {tot }} / M_{\mathrm{b}}^{\text {SMNS }}$ is closer to the value for the BL model. All models have a total mass 4-8\% larger than the maximum possible for uniformly rotating models. We therefore expect that the mergers result in short lived remnants, and not in prompt collapse (compare [60]). The three models allow to determine separately the influence of the EOS and of the mass. Their parameters are summarized in Table II.

Initial data are computed with the publicly available LORENE code [62,63], assuming an irrotational binary on a
TABLE II. Initial data parameters. $M_{\mathrm{b}}^{\mathrm{SMNS}}$ is the maximum baryonic mass for a uniformly rotating NS, $M_{\mathrm{b}}^{\text {tot }}$ is the total baryonic mass of the system, $M_{\mathrm{g}}$ and $R_{c}$ are gravitational mass and circumferential radius of each star at infinite separation, $f_{0}$ is the initial orbital frequency, $d$ the initial proper separation, and $\Lambda / M_{g}^{5}$ is the dimensionless tidal deformability (see [61]).

\begin{tabular}{lccc}
\hline \hline Model & BL-1.35 & GM3-1.31 & GM3-1.35 \\
\hline$M_{\mathrm{b}}^{\text {tot }}\left[M_{\odot}\right]$ & 2.95 & 2.83 & 2.92 \\
$M_{\mathrm{b}}^{\text {tot }} / M_{\mathrm{b}}^{\text {SMNS }}$ & 1.04 & 1.05 & 1.08 \\
$M_{\mathrm{g}}\left[M_{\odot}\right]$ & 1.35 & 1.31 & 1.35 \\
$M_{\mathrm{g}} / R_{c}$ & 0.162 & 0.144 & 0.148 \\
$f_{0}[\mathrm{~Hz}]$ & 281 & 278 & 282 \\
$d[\mathrm{~km}]$ & 58 & 57 & 58 \\
$\Lambda / M_{g}^{5}$ & 492 & 957 & 793 \\
\hline \hline
\end{tabular}

quasicircular orbit. We note that the initial absence of radial velocity leads to some eccentricity during the evolution. The initial proper separation of $57-58 \mathrm{~km}$ allows the completion of 5-6 orbits.

All the simulations discussed in this paper use the WhiskyThermal code $[64,65]$ for the evolution of the hydrodynamic equations in general relativity. The spacetime is evolved with the publicly available McLachlan code, which implements the BSSNOK formulation of the field equations [66-68]. Both parts are coupled together within the publicly available Einstein Toolkit [69]. The latter also includes the Carpet code [70], which we use for adaptive mesh refinement.

The general relativistic hydrodynamic equations are evolved using high resolution shock-capturing schemes based on the flux-conservative "Valencia" formulation [71]. We compute fluxes using the HLLE [72] approximate Riemann solver, which uses primitive variables reconstructed at cell interfaces with the piecewise parabolic method. Finally we employ an artificial atmosphere with a floor value of $\rho_{\text {atmo }}=6.2 \times 10^{4} \mathrm{~g} \mathrm{~cm}^{-3}$ as well as zero velocity and temperature.

For the mesh refinement, we use fixed-size moving boxes during inspiral. The two refinement levels with the highest resolutions consist of nested cubes following the two NSs. Four additional coarser levels consist of larger, nonmoving cubes. At merger, we switch to fixed mesh refinement. The cube of highest resolution has a half-diameter of $26 \mathrm{~km}$, covering the remnant and the innermost part of the accretion disk. The finest grid resolution is $d x=186 \mathrm{~m}$, which means the NSs are resolved with $\sim 53-60$ points per coordinate radius, depending on the model. The outer boundary in our simulations is located at $1178 \mathrm{~km}$. In order to save computational resources, we evolve the system with reflection symmetry across the orbital plane.

\section{MERGER AND POSTMERGER DYNAMICS}

In the following, we describe the dynamics of inspiral and merger, and investigate the evolution of the remnant 
TABLE III. Outcome of our BNS mergers. $M_{\mathrm{BH}}$ and $J_{\mathrm{BH}}$ are black hole mass and angular momentum $5 \mathrm{~ms}$ after formation (only for collapsing models). $M_{\mathrm{blk}}$ and $R_{\mathrm{blk}}$ are bulk mass and bulk radius (see text for definitions), while $\nu_{\mathrm{c}}$ and $\nu_{\max }$ denote the remnants central and maximum rotation rates, all computed $12 \mathrm{~ms}$ after merger. $f_{\text {merge }}$ is the gravitational wave instantaneous frequency at the time of merger, $f_{\mathrm{pm}}$ is the frequency of the maximum in the postmerger part of the gravitational wave power spectrum, and $f_{10}$ is the amplitude-weighted average instantaneous frequency during the first $10 \mathrm{~ms}$ after merger (see [73]). $M_{\text {disk }}^{\text {alt }}$ is the mass at densities below $10^{-13} \mathrm{~g} / \mathrm{cm}^{3}, M_{\text {disk }}$ is the mass outside a coordinate sphere of volumetric radius $r=30 \mathrm{~km}, M_{\mathrm{fb}}$ is the bound mass outside coordinate radius $r>60 \mathrm{~km}$, all measured $12 \mathrm{~ms}$ after merger. The value in brackets denotes the mass outside the apparent horizon at $5 \mathrm{~ms}$ after $\mathrm{BH}$ formation. Finally, $M_{\mathrm{ej}}$ and $v_{\infty}$ are our estimates for the total ejected mass and the average expansion velocity at infinity.

\begin{tabular}{lccc}
\hline \hline Model & BL 1.35 & GM3 1.31 & GM3 1.35 \\
\hline$M_{\mathrm{BH}}\left[M_{\odot}\right]$ & $\ldots$ & $\ldots$ & 2.54 \\
$J_{\mathrm{BH}} / M_{\mathrm{BH}}^{2}$ & $\ldots$ & $\ldots$ & 0.65 \\
$M_{\mathrm{blk}}\left[M_{\odot}\right]$ & 2.52 & 2.40 & 2.52 \\
$M_{\mathrm{blk}} / R_{\mathrm{blk}}$ & 0.30 & 0.26 & 0.29 \\
$\nu_{\mathrm{c}}[\mathrm{kHz}]$ & 0.79 & 0.68 & 0.72 \\
$\nu_{\text {max }}[\mathrm{kHz}]$ & 1.59 & 1.26 & 1.43 \\
$f_{\text {merge }}[\mathrm{kHz}]$ & 1.87 & 1.72 & 1.69 \\
$f_{\mathrm{pm}}[\mathrm{kHz}]$ & 3.17 & 2.74 & 2.89 \\
$f_{10}[\mathrm{kHz}]$ & 3.09 & 2.66 & 2.79 \\
$M_{\text {disk }}\left[M_{\odot}\right]$ & 0.086 & 0.077 & $0.057(0.050)$ \\
$M_{\mathrm{disk}}^{\text {alt }}\left[M_{\odot}\right]$ & 0.136 & 0.135 & 0.097 \\
$M_{\mathrm{fb}}\left[M_{\odot}\right]$ & 0.062 & 0.046 & 0.040 \\
$M_{\mathrm{ej}}\left[10^{-2} M_{\odot}\right]$ & 0.62 & 0.14 & 0.10 \\
$v_{\infty}[c]$ & 0.17 & 0.13 & 0.10 \\
\hline \hline
\end{tabular}

properties. The quantitative results discussed below are summarized in Table III.

All three BNS models complete $\sim 5-6$ orbits before merger. Fig. 3 shows the proper separation as function of the orbital phase. We find a residual eccentricity which is typical for initial data obtained with the quasicircular approximation (see Sec. III) without corrections for the radial velocity of the inspiral. Therefore, we do not try to make quantitative statements about tidal effects on the orbital dynamics. Qualitatively, we find a longer inspiral for model $B L-1.35$. Eccentricity aside, this agrees with the theoretical expectation because the NSs are more compact than those of the GM3 models.

None of our models forms a BH directly at merger. Consequently, the threshold for prompt collapse (in the equal mass case) is above a total gravitational mass of $2.7 M_{\odot}$ for both EOS. For model GM3 - 1.35, a BH is formed $\sim 14 \mathrm{~ms}$ after merger (final mass and angular momentum given in Table III). For the other two models, $G M 3-1.31$ and $B L-1.35$, the remnants survive at least $5 \mathrm{~ms}$ longer, at which point our simulations end. A strong

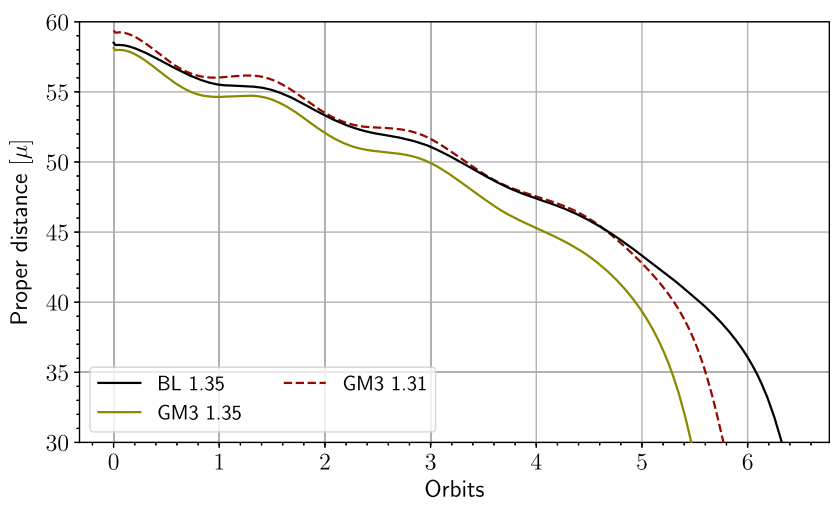

FIG. 3. Proper separation between the NS barycenters during the inspiral as function of orbital phase. The separation is given in units of reduced mass $\mu=M_{g}^{1} M_{g}^{2} /\left(M_{g}^{1}+M_{g}^{2}\right)$. The barycenters and orbital phases are computed using simulation coordinates.

influence of EOS and mass on the lifetime is not surprising, since our systems are in the hypermassive mass range.

Snapshots of the density distribution after merger are given in Figs. 4 and 5 for all three models. One can observe the formation of a disk in all cases. It is worth noting that the disk keeps growing until a $\mathrm{BH}$ forms. The cause might be a complex fluid flow in the outer layers of the strongly deformed remnant, involving rotational vortices. Such a mechanism was investigated in detail for a different model in [74]. Also the temperature evolution (based on the simplified prescription for the thermal pressure) is qualitatively similar to the one in [74], as shown in Appendix.

In order to quantify the properties of the disk, we first need to discuss its structure, in particular the lack of a clear distinction between a HMNS and the surrounding disk. Figure 6 shows one example of the disk structure. Ideally, we would like to distinguish between remnant and disk mass in a meaningful way which is also easy to extract from the simulation data. From the available data, we can extract the total mass outside a given cutoff radius or below a certain density threshold. However, such measures depend strongly on the chosen cutoff values. This can be seen from the density isosurfaces shown in Fig. 6 and the enclosed mass fractions (given in the legend).

One meaningful definition of a disk (at a given time) is the region which would not be swallowed if the central remnant would suddenly collapse to a $\mathrm{BH}$. This will depend mainly on mass and spin of the BH. For model $G M 3-1.35$, we picked a cutoff radius such that the mass outside shortly before collapse roughly corresponds to the mass remaining outside of the apparent horizon shortly after collapse. Since we expect similar properties of the final $\mathrm{BH}$ for the other models, we use the same definition for the disk mass $M_{\mathrm{d}}$ also for these. To reduce gauge ambiguities, we specify the cutoff radius based on the proper volume enclosed inside the coordinate sphere. The chosen cutoff volume is that of an Euclidian sphere with a 


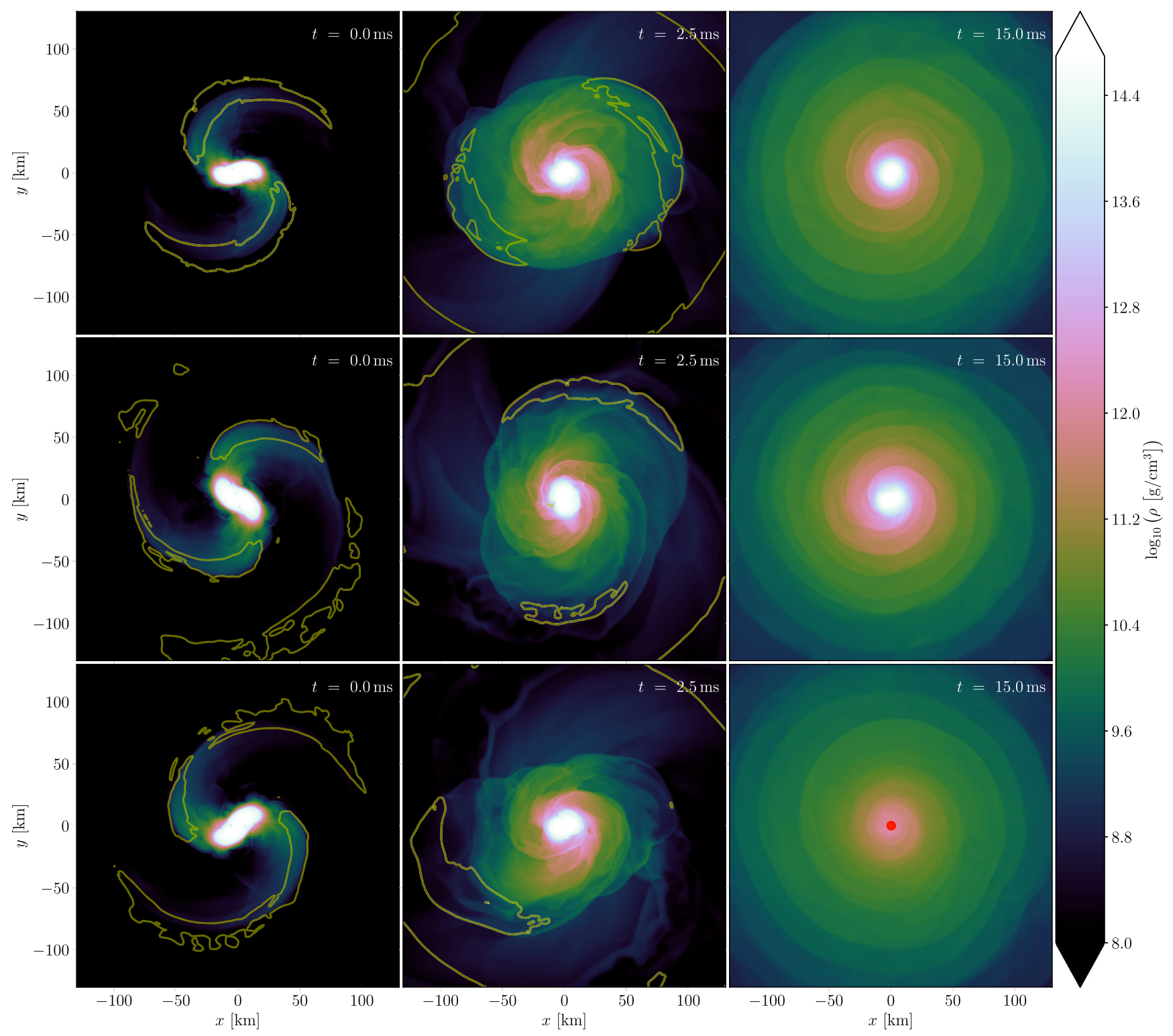

FIG. 4. Rest-mass density evolution in the equatorial plane for the three simulations $B L-1.35$ (top), GM3 - 1.31 (middle) and GM3 - 1.35 (bottom). The yellow contour lines highlight the unbound ejected matter. The snapshots are taken at three different phases of the evolution: on the left at the time of merger, in the middle $2.5 \mathrm{~ms}$ after merger, and on the right $15 \mathrm{~ms}$ after merger. The apparent horizon of the newly formed $\mathrm{BH}$ for the GM3 - 1.35 model is highlighted with a red circle.

radius of $30 \mathrm{Km}$, the corresponding coordinate radius is marked in Fig. 6 with a white circle.

For comparison with [75], we also employ an alternative measure for the disk mass, $M_{\mathrm{d}}^{\text {alt }}$, defined as the total mass below a density of $10^{13} \mathrm{~g} / \mathrm{cm}^{3}$. As shown in Fig. 6, this definition cuts the disk much closer to the remnant. In particular, it includes material around the rotation axis, which will be swallowed when the $\mathrm{BH}$ is formed. At least for our models, the resulting disk mass is not a good estimate for the disk mass remaining after collapse. Similarly, it might depend more strongly on the exact structure of the remnant. This is relevant for the interpretation of [75], where the measure is used as upper limit for mass ejection from the disk for the cases where no $\mathrm{BH}$ forms during the simulation.

The time evolution of the disk masses is shown in Fig. 7. We can see that the two disk definitions differ significantly, by a factor $\approx 1.6$. Note that the error of the density based estimate will increase when the true value decreases since the estimate includes some material that will collapse together with the remnant. As mentioned before, the disk is increasing gradually before $\mathrm{BH}$ formation. Such a mass expulsion might delay the collapse of HMNSs and also contribute to the seemingly unpredictable amplitude evolution of GW signals from HMNSs. 


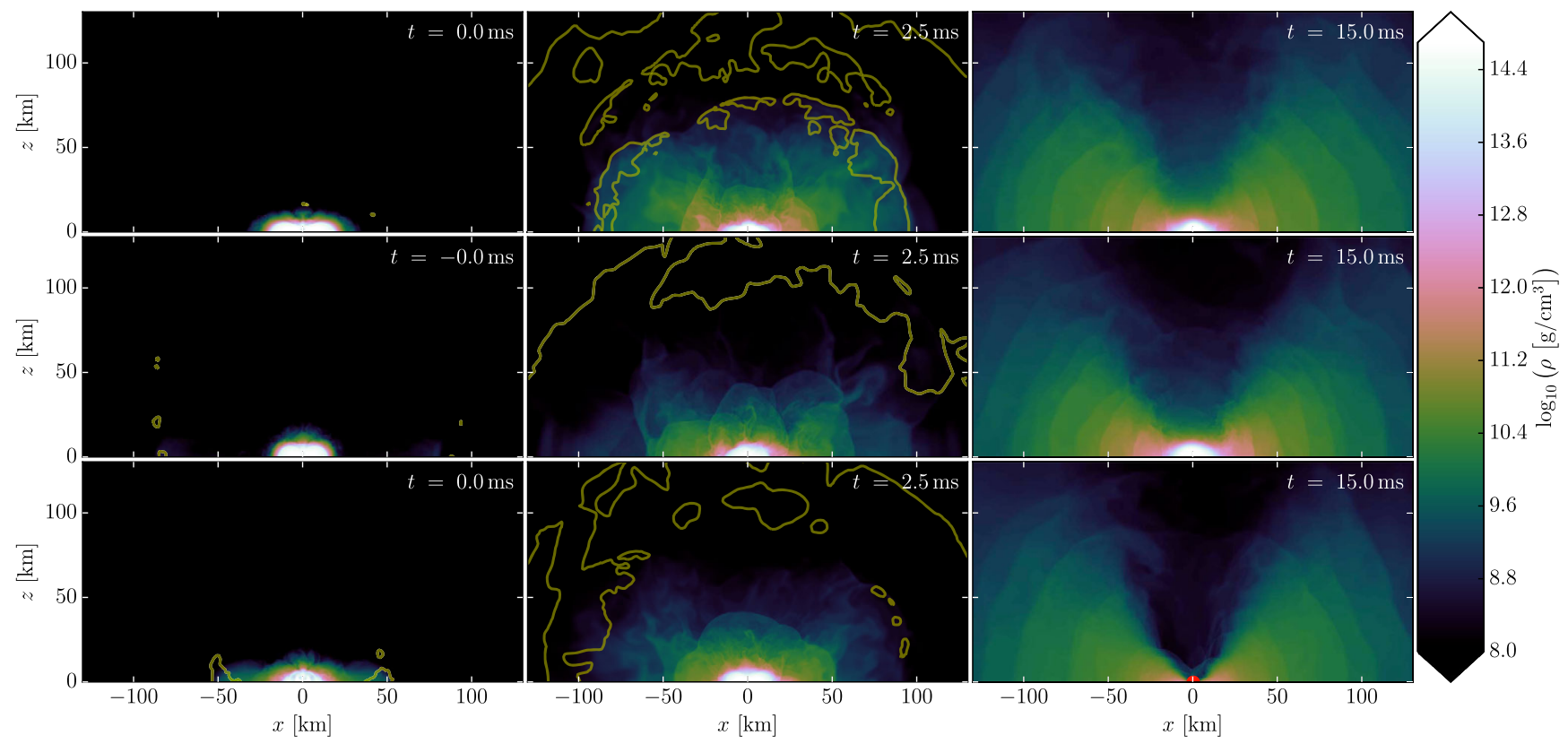

FIG. 5. Same as Fig. 4, but showing a cut in the meridional plane.

Note that the values we provide in Table III refer to a specific time. Although the growth rate slows down, the disk masses are not constant. We stress that values for the disk mass extracted from numerical simulations should be regarded only as ballpark figures because of the ambiguities arising from extraction time and disk mass definition.

Comparing our results for disk and ejecta masses to Fig. 1 in [75], we find that models $B L-1.35$ and GM3-1.35 are outliers to the proposed correlation.

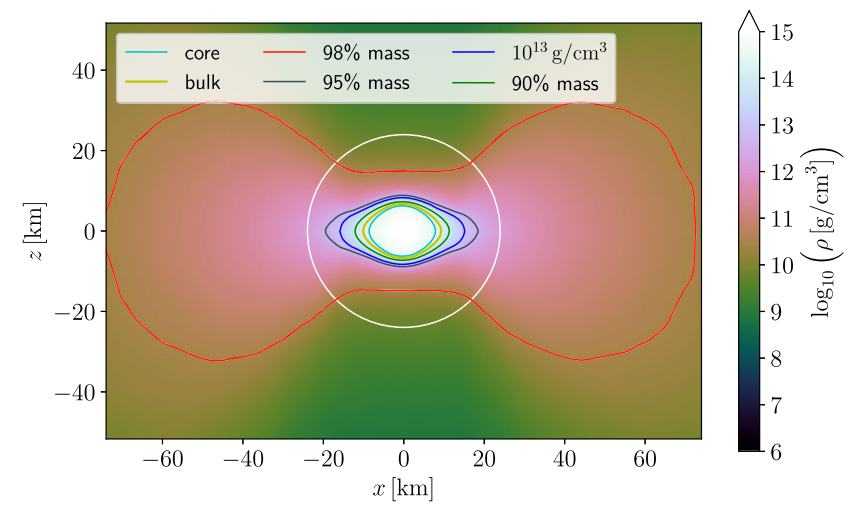

FIG. 6. Structure of the disk and embedded remnant for model $G M 3-1.31$, at the end of the simulation. The mass density is shown as color plot. In order to remove contributions from oscillations, we average in time over the last $4 \mathrm{~ms}$. The white circle marks the cutoff (coordinate) radius used to define the disk mass $M_{\mathrm{d}}$. The density cutoff $10^{13} \mathrm{~g} / \mathrm{cm}^{3}$ used for the alternative definition $M_{\mathrm{d}}^{\text {alt }}$ is marked by the blue contour. The other contours mark isodensity surfaces containing 90,95 , and $98 \%$ of the total baryonic mass, and those corresponding to remnant bulk and core (see text).
Although the disk masses in [75] for noncollapsing models were extracted somewhat later than ours, our disk mass $M_{\mathrm{d}}^{\text {alt }}$ for $B L-1.35$ is larger and still increasing at the end of our simulation. For $G M 3-1.35$, the mass remaining outside the $\mathrm{BH}$ is lower and still decreasing. The deviation might be due to the model selection in [75], or due to the numerical errors of our simulation. Further high-resolution studies are needed to estimate the latter. Nevertheless, our results indicate that the ambiguities in disk mass definition and extraction time add a factor $\approx 2$ to the disk mass error.

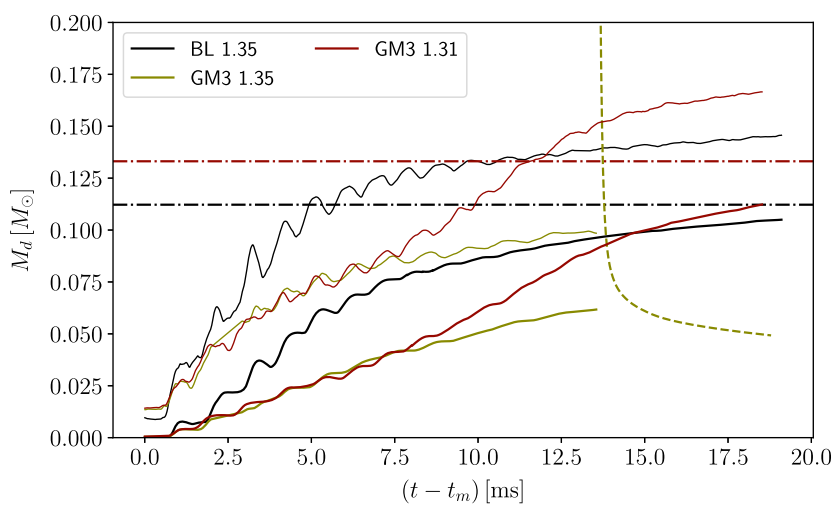

FIG. 7. Time evolution of disk mass for our simulations. The solid thick lines show the disk mass measure $M_{\mathrm{d}}$, and the thin solid lines the alternative measure $M_{\mathrm{d}}^{\text {alt }}$. After the $\mathrm{BH}$ formation for model GM3 - 1.35, we show the mass outside the apparent horizon instead (dashed line). The rapid initial decrease corresponds to the rapid initial growth of the apparent horizon. The horizontal lines mark a disk mass for which the remaining remnant mass is at the upper limit for a SMNS (the two GM3 models are indistinguishable due to almost identical total mass). 
We also point out that the mass fraction which is ejected from the disk for a given model is another important unknown not considered in [75]. Studies modeling said fraction find typical values in the range $10-50 \%$ with different methods and assumptions (see e.g., [8,76-81]). If, e.g., only $1 / 5$ of the disk mass can be ejected, none of the simulations would reach the required ejecta mass. The above references also indicate that a reliable measure for the mass of the disk might be required, but not sufficient, because disk structure and neutrino irradiation, as well as magnetic fields, also play an important role for the ejected mass. In order to constrain the EOS this way, a reliable modeling of winds ejected from the disk is required in addition to robust kilonova light curve models.

For both measures, the disk mass is initially larger for model $B L-1.35$ than for the GM3 models. Interestingly, the growth rate of the disk increases for model GM3-1.31, which has the largest disk mass at the end of the simulations. The reason is unknown, but might be related to a growing $m=1$ oscillation.

It is natural to ask if the mass of the remnant itself, excluding the disk, is still in the hypermassive range. Matter which would not fall into the black hole in case of sudden collapse should orbit close to Keplerian speed. Hence it should have only little influence on the stability of the remnant at a given time. In Fig. 7, we also show the minimum disk mass such that the remnant is supramassive. We find that the remnant for model $G M-1.35$ is still a HMNS, for both definitions of the mass. For the other two models, the remnant is a HMNS according to our disk measure $M_{\mathrm{d}}$, but a SMNS according to $M_{\mathrm{d}}^{\text {alt }}$.

We now turn to discuss the structure of the HMNS remnants. Besides the mass, one of the properties most relevant for delaying the collapse is the rotation profile. Figure 8 shows the angular frequency as a function of radius $11 \mathrm{~ms}$ after merger. In all cases, we observe a slowly rotating core, while the equatorial bulge approaches the

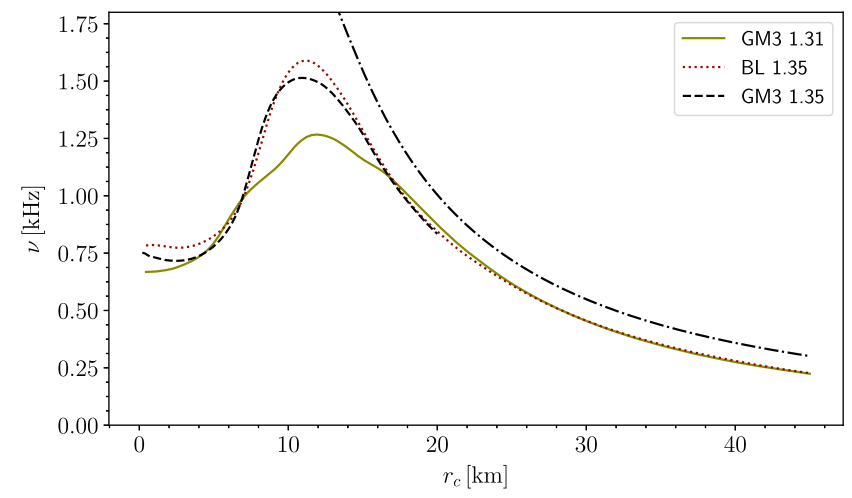

FIG. 8. Rotation profile of the remnant for our models $11 \mathrm{~ms}$ after merger. $r_{c}$ is the circumferential radius, and $\nu$ the rotation frequency as observed from infinity. The dash-dotted curve represents the frequency for a test particle on a prograde circular orbit.
Keplerian rotation rate. We interpret the residual difference in the disk as the contribution from the pressure gradient. The shape of the rotation profile seems to be a generic feature of merger remnants, which was observed in several studies [73,74,82-85] for many different models.

We note that the profiles for model GM3-1.31 and GM3 - 1.35 differ significantly, despite the similar mass. The reason is unclear. However, the HMNSs are close to collapse and it is not surprising if the exact evolution is very sensitive to small changes of the initial parameters. Model $G M 3-1.31$ also develops a stronger $m=1$ perturbation, which might be related.

As discussed in [74], the radial mass distribution in the slowly rotating core can be approximated well by the core of an nonrotating NS (TOV solution). Since the mass of TOV solutions is bounded, this defines a critical mass of the remnant core. In [73], we proposed that the HMNS collapses when the TOV core equivalent reaches the maximum mass. This was confirmed for two models studied in [73].

Before comparing the conjecture to our results, we briefly summarize the gauge independent measures introduced in [74] for this purpose. They are based on the proper volume and baryonic mass enclosed inside surfaces of constant mass density. From volume and mass, we derive a radius and a compactness measure for each isosurface. Further, we define the bulk of a star as the region inside the single isosurface which is most compact according to the new measure. This allows us to unambiguously define the TOV core equivalent of the merger remnant as the TOV solution for which volume and mass of the bulk coincide with mass and volume of some isosurface inside the remnant. Finally, we define the remnant core as the interior of the isosurface with the density identical to the density on the TOV core equivalent's bulk surface. Inside this core, the radial mass distribution of equivalent TOV and remnant agree well. For further details, see [74]. Bulk and core of the remnant for model GM3 - 1.31 are shown in Fig. 6 for comparison.

The time evolution of the TOV core equivalents is shown in Fig. 9. We find that model GM3 - 1.35 indeed collapses once the bulk mass of the core equivalent TOV exceeds the maximum. We note that the remnant for model GM3 - 1.35 is not accreting matter before collapse, but, on the contrary, sheds mass into the disk. The observed collapse could be caused by internal rearrangement of the HMNS structure and/or loss of the angular momentum via the matter migrating into the disk.

For the other models, the bulk mass is still well below the maximum at the end of the simulation. Extrapolating the core evolution, we estimate that the remnants for models $B L-1.35$ and $G M 3-1.31$ might survive for another $\approx 10 \mathrm{~ms}$.

We stress that our discussion of remnant lifetimes is purely qualitative. We found that the outcome of our 


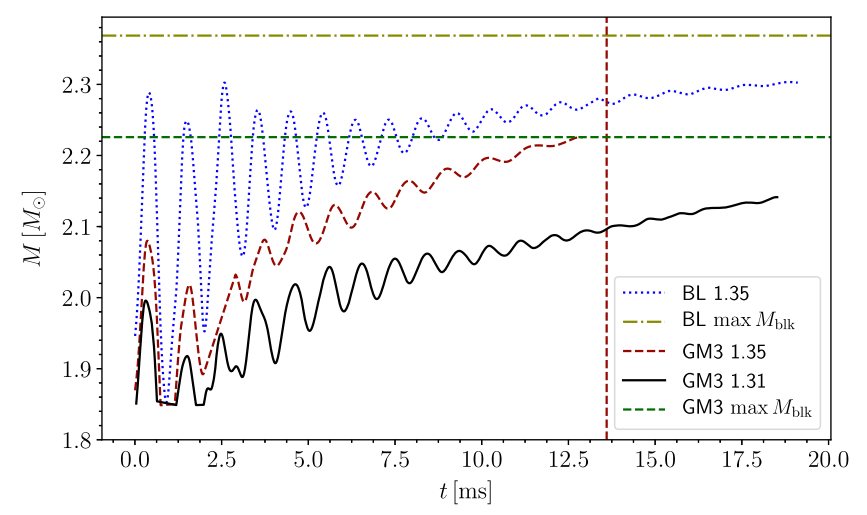

FIG. 9. Time evolution of the TOV solutions equivalent to the remnant cores (see text) for our simulations. The curves show the bulk mass of the TOV solutions, and the horizontal lines mark the maximum bulk mass for TOV sequences of the given EOS. The vertical line marks the $\mathrm{BH}$ formation.

simulations is very sensitive to mass and EOS, likely because the system is close to collapse. Therefore we also expect the lifetime to be sensitive to numerical errors, and, most importantly, the physical viscosity. In [86], it was demonstrated that prescribing an effective alpha viscosity affects the evolution of the rotation profile. Such an effective viscosity could be caused by magnetic field amplification processes, although it is difficult to model the magnetic field on small scales numerically (see [87]).

Last but not least, we discuss the prospects for producing a SGRB. In particular, we consider the scenario of a $\mathrm{BH}$ embedded in a disk, with a strong ordered magnetic field around the $\mathrm{BH}$ rotation axis. Regardless of the disk mass definition, all our models develop a significant disk. For model GM3 - 1.35, a BH surrounded by a disk is formed during the simulation. For the other models, we expect collapse within tens of ms. Moreover, the disk mass is still growing at the end of the simulations. It is therefore likely that the disk mass is not significantly reduced when the HMNSs finally collapse.

We did not include magnetic fields in our simulations and, therefore, do not discuss the prospects for strong magnetic fields in our models. For a general discussion, we refer to [73,88-90] instead.

Since baryon pollution might suppress the formation of a jet, the baryon density along the $\mathrm{BH}$ axis is an important aspect with regard to SGRBs. Therefore, we computed the rest mass density along the rotation axis, averaged between $30-50 \mathrm{Km}$ above the equatorial plane (see Fig. 10). We note that the density at any time after merger is more than two orders of magnitude above the artificial atmosphere density, which can be safely ignored.

The time evolution of the density is shown in Fig. 5. During the HMNS lifetime, all models exhibit a comparable density of $\approx 10^{9} \mathrm{~g} / \mathrm{cm}^{3}$. For model GM3 -1.35 , the density decreases by almost two orders of magnitude within $5 \mathrm{~ms}$ as soon as the $\mathrm{BH}$ is formed. Those findings are

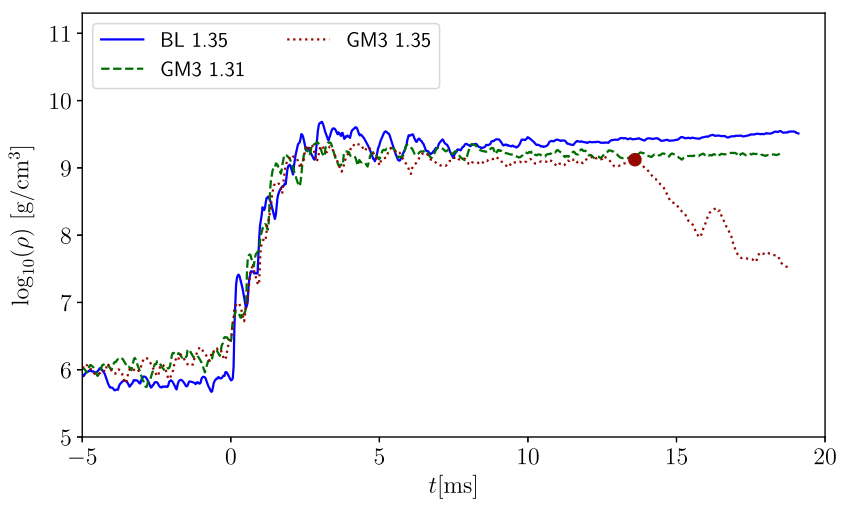

FIG. 10. Average rest mass density along the rotation axis above the remnant. The average is taken between a distance 30 and $50 \mathrm{~km}$ from the origin. The density around $10^{6} \mathrm{~g} / \mathrm{cm}^{3}$ during the inspiral is due to matter ejected by numerical artifacts at the NS surfaces. For comparison, the density of the artificial atmosphere used in our simulations is $6 \times 10^{4} \mathrm{~g} / \mathrm{cm}^{3}$.

similar to the results obtained for different models in a previous work [73]. Our results are also relevant for studies on the possibility of low-mass BNS as the central engine for SGRBs (e.g., [90-92]). For further discussions, also compare [93-96].

\section{MASS EJECTION AND KILONOVA}

We now turn to discuss the ejection of matter during and after merger. The mass, geometrical distribution, expansion velocity, and composition of this outflow are the key ingredients to predict the associated electromagnetic emission, in particular the kilonova signal.

Matter can be expelled dynamically during or shortly after merger by different mechanisms. Tidal forces during merger eject cold, neutron-rich matter in the orbital plane, which can however be heated when faster tidal ejecta collide with slower ones. Shock waves formed during merger can liberate matter from the remnant surface (breakout shocks). Breakout shocks are thought to yield less neutron-rich matter, which can also be launched outside the orbital plane, including the rotation axis. Oscillations of the remnant can cause waves in the surrounding debris material which can also steepen into shocks. Since the r-process nucleosynthesis and the resulting kilonova signal depend on the thermal history, the electron fraction, and the velocity of the ejecta, it is very important to identify the different ejecta components and the corresponding ejection mechanisms (see [97] for a discussion).

To determine if a fluid element will be dynamically ejected, we adopt the geodesic criterion $u_{t}<-1$, where $u$ is the 4-velocity (see $[73,83]$ for further explanation). The snapshots shown in Figs. 4 and 5 also mark the regions with unbound matter. We observe typical spiral patterns of tidal ejecta in the orbital plane as well as other ejecta in all directions, likely caused by shocks. 


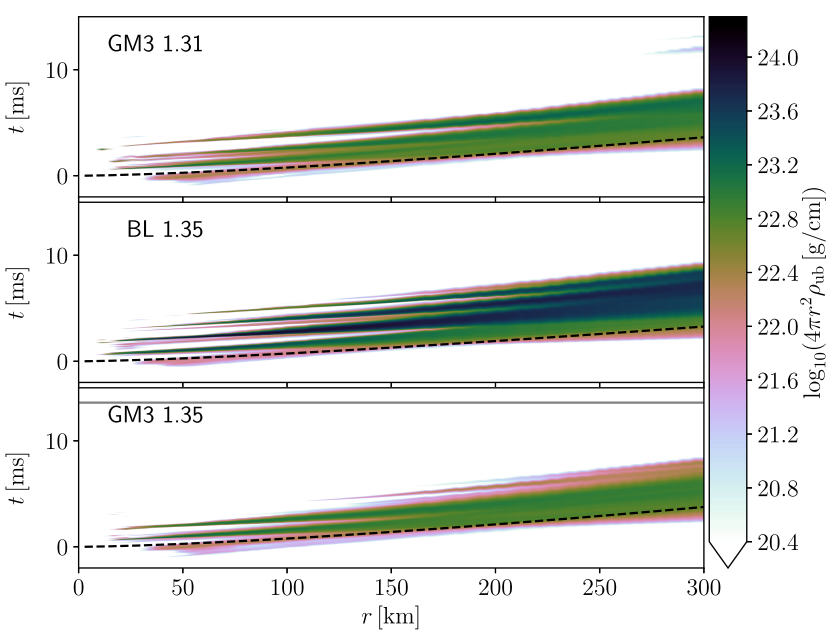

FIG. 11. Radial distribution of unbound matter as a function of time. The logarithmic color scale corresponds to the unbound mass per coordinate radius. The black dashed line represents the trajectory of a radially outgoing test mass with velocity $v_{\infty}$ from Table III. The trajectory has been estimated using the Newtonian potential of a mass equal to the ADM mass at the end of the simulation.

In Fig. 11, we show the time evolution of the radial distribution of unbound matter for the three BNS models. One can clearly distinguish several waves of ejected matter, which merge at larger radii. Only the first wave is launched around the time of merger. The subsequent waves are likely related to the oscillations of the remnant interacting with the surrounding matter.

In order to quantify the matter ejection, we monitor the flux of unbound matter through several spherical surfaces, at radii 50, 100, 200, 500, $700 M_{\odot}$. For each, we integrate the flux of unbound mass in time, and take the maximum over the different surfaces as best estimate for the true amount of dynamically ejected matter (see $[73,83]$ ). The results for all models are given in Table III.

Further, we compute an average final expansion velocity from

$$
\bar{u}_{t}=\frac{1}{M_{e}} \int W \rho_{u} u_{t} \mathrm{~d} V \quad M_{e}=\int W \rho_{u} \mathrm{~d} V
$$

where $\rho_{u}$ is the rest-frame (baryonic) mass density of unbound matter, $W$ its Lorentz factor, and $\mathrm{d} V$ the proper 3 -volume element. The integration is carried out over the region $r>150 \mathrm{Km}$, outside the strongly dynamic region, and at the time where $M_{e}$ becomes maximal. We obtain an average Lorentz factor $\bar{W}_{\infty}=-\bar{u}_{t}$, which the matter will approach at infinity. The corresponding average expansion velocity $v_{\infty}$ is given in Table III. Our values fall in the range found in simulations of other BNS systems $[60,73,83,98-$ 100]. We estimate that thermal effects might increase the

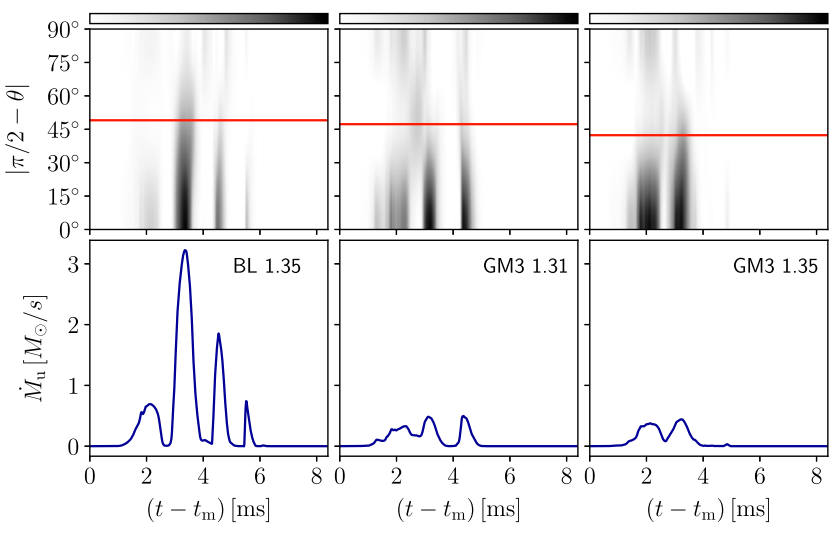

FIG. 12. Time evolution of the unbound matter flux through a spherical surface at $r=148 \mathrm{Km}$. Top panels: polar distribution of the flux reconstructed from the multipole moments up to $l=4$. The orbital plane corresponds to $0^{\circ}$ and the orbital axis to $90^{\circ}$. The color scale is linear, ranging from zero (white) to the maximum flux for the given model (black). The horizontal line marks the angle below which $90 \%$ of the matter is ejected during the whole evolution. Bottom panels: total flux through the spherical surface, showing the contribution of the separate ejecta waves.

given expansion velocity by up to $20 \%$ (based on comparing Bernoulli and geodesic criteria for our simulation data).

Model $B L-1.35$ results in a significantly larger amount of unbound material compared to the two models with GM3 EOS. For model $B L-1.35$, we also find larger fluctuations of the central density and a stronger modulation of the GW frequency, which will be discussed in Sec. VI. Stronger quasiradial oscillations of the remnant are one possible explanation for the higher ejecta mass from model $B L-1.35$. The stronger oscillations could be related to the larger compactness of the initial NS of this model.

To further investigate the main ejection mechanism, we compute the mass flux corresponding to the different waves, shown in the lower panels of Fig. 12. The mass ejection for model $B L-1.35$ is indeed dominated by the waves launched after merger, while the tidally ejected first wave contributes little. We note that the result might change for systems with large mass ratio, which typically produce more tidal ejecta.

Next, we investigate the polar distribution of ejected matter. During the simulations, we compute multipole moments (up to $l=4$ ) of the unbound matter flux on the aforementioned spherical surfaces. From this data, we reconstruct an approximate distribution, which is shown in the upper panels of Fig. 12. We find that $90 \%$ of the matter is ejected at angles less than $50^{\circ}$ from the orbital plane.

We can now estimate the kilonova signal that would be produced by these dynamical ejecta (ignoring potential later outflows). For this, we apply the simple analytical model by [101] (using the same parameter value $\alpha=1.3$ ), and assume that all the ejecta material is involved in the 
TABLE IV. Order of magnitude estimates for the kilonova signal caused by the dynamical ejecta, computed from the analytic approximations given in [101], for two different assumptions about the opacity $\kappa$. Using ejecta mass and expansion velocities shown in Table III, we obtain the time $t_{\text {peak }}$ where peak luminosity $L_{\text {peak }}$ is reached, and the corresponding temperature $T_{\text {peak }}$. We also provide the maximum-intensity wavelength $\lambda_{\text {peak }}$ of the respective black-body spectrum.

\begin{tabular}{lcccrr}
\hline \hline Model & $\begin{array}{c}\kappa \\
{\left[\mathrm{cm}^{2} / \mathrm{g}\right]}\end{array}$ & $\begin{array}{c}t_{\text {peak }} \\
{[\text { days }]}\end{array}$ & $\begin{array}{c}L_{\text {peak }} \\
{\left[10^{40} \mathrm{erg} / \mathrm{s}\right]}\end{array}$ & $\begin{array}{c}T_{\text {peak }} \\
{\left[10^{3} \mathrm{~K}\right]}\end{array}$ & $\begin{array}{r}\lambda_{\text {peak }} \\
{[\mathrm{nm}]}\end{array}$ \\
\hline$B L-1.35$ & 0.5 & 0.7 & 21 & 7.8 & 371 \\
& 10 & 3.0 & 3.0 & 2.3 & 1276 \\
GM3-1.35 & 0.5 & 0.3 & 7.8 & 11.0 & 263 \\
& 10 & 1.5 & 1.1 & 3.2 & 906 \\
GM3-1.31 & 0.5 & 0.4 & 10 & 10.2 & 284 \\
& 10 & 1.6 & 1.5 & 3.0 & 979 \\
\hline \hline
\end{tabular}

r-process nucleosynthesis (for more on kilonova models see $[8,102-105])$. Besides ejected mass and expansion velocity, the analytical estimates depend strongly on the opacity, which depends mainly on the amount of lanthanides produced. The latter depends on the initial neutron fraction of the ejecta. Our simulations do not include neutrino radiation and employ an EOS assuming beta equilibrium and simplified treatment of thermal effects. Therefore, we cannot predict the ejecta composition. Instead, we provide estimates for a plausible opacity range. In detail, we consider lanthanide-poor ejecta with opacity $0.5 \mathrm{~cm}^{2} / \mathrm{g}$, and lanthanide-rich ejecta with opacity $10 \mathrm{~cm}^{2} / \mathrm{g}$ (see [106] and references therein).

The results are given in Table IV. The unknown opacity introduces factors $4.5,7$, and 3.5 to the uncertainties of peak time, peak luminosity, and temperature, respectively. Although a simultaneous measurement of all three would fix opacity, ejecta mass, and expansion velocity within the analytic model, the model uncertainties would likely not allow to distinguish between the BL and GM3 EOS for the given mass $1.35 M_{\odot}$. However, the large differences between the two EOS obtained within the approximation indicate that it should be possible to distinguish between the two EOS considered here if the kilonova signal could be predicted more accurately. For the lanthanide-poor opacity, the peak time differs by a factor $\approx 2$ between BL and GM3, the luminosity by a factor $\approx 3$, and the temperature by 1.4 .

It is instructive to compare the potential kilonova signal of our models $B L-1.35$ and $G M 3-1.35$ to the optical counterpart AT2017gfo (see [3]) observed for the GW event GW170817. We emphasize that there is no reason to expect agreement, since the chirp mass inferred for GW170817 is $1 \%$ larger, and because the mass ratio is not well constrained. The evolution of luminosity and spectrum of AT2017gfo can be fitted by a kilonova model consisting of independent components caused by distinct ejecta waves with different opacities, masses, and velocities [60,106,107]. The model in [107] includes a "blue" component caused by low-lanthanide ejecta with mass $\approx 0.025 M_{\odot}$, expanding at $\approx 0.3 c$. Note the high velocity is also inferred from the fact that the observed spectrum is essentially featureless. Similar results are derived from the kilonova models in [106]. Given the lower masses and lower velocities (see Table III), we conclude that models $B L-1.35$ and $G M 3-1.35$ are unlikely to produce a counterpart similar to the blue component of AT2017gfo, even when assuming lowlanthanide ejecta. We also note that our ejecta are dominantly shock-driven and mostly equatorial, and it is difficult to guess their composition. Before becoming unbound, they orbit close to the remnant, while being irradiated by its neutrino emission. Further, they are heated by multiple shocks, as shown in Fig. 15 in Appendix.

In addition to the blue component discussed above, the kilonova signal AT2017gfo shows also a "red" component, peaking in the IR band several days after merger (see, e.g., [107]). The latter is modeled in [107] by a very massive ejection of matter $\left(\approx 0.04 M_{\odot}\right)$ which is expanding more slowly $(\approx 0.1 c)$. The likely explanation for the high mass is that the red component of AT2017gfo was caused, at least partially, by winds expelled from the disk (and possibly a long-lived remnant) due to neutrino radiation and magnetic fields (see, e.g., [108-110]).

Since our models possess massive disks, one should expect a contribution to the kilonova signal as well. The mass ejected as winds is, however, difficult to model, and therefore we can make no predictions about the corresponding light curves. Instead, we discuss the implications if our models were to eject $0.04 M_{\odot}$ in form of winds, similar to AT2017gfo. The first important aspect is whether the remaining mass could still form a $\mathrm{BH}$, and how long it would take. Comparing the remaining mass to the maximum mass of supramassive stars (see Fig. 7), we find that all three models would still be in the hypermassive mass range, and therefore form a $\mathrm{BH}$ on short timescales $<1 \mathrm{~s}$. The next question is how much of the material can originate from the disk. Comparing to Fig. 7, we find that around half of the disk mass $M_{\mathrm{d}}$ would need to be ejected for model $B L-1.35$ if the wind originates entirely from the disk. From the discussion in Sec. IV, it seems likely that a BH is formed within $\lesssim 20 \mathrm{~ms}$ (compare Fig. 7). Therefore, we assume that winds originating directly from the surface will not contribute much. For model $G M-1.35,75 \%$ of the disk would need to be ejected. Since the remnant is a $\mathrm{BH}$, it cannot provide additional mass. The situation for model GM3 - 1.31 falls between the other two.

We caution that the above discussions are only qualitative and model dependent. For example, if the merger produced a successful (or choked) jet accompanied by a wide-angle and mildly relativistic cocoon (e.g., [111,112]), it could alter the contribution of the shock-driven ejecta to 
the blue kilonova component; however, the energy of the latter $\left(\sim 10^{51} \mathrm{erg}\right)$ is orders of magnitude larger than the observed energies for short GRBs $\left(\sim 10^{49}-10^{50}\right.$ [113]) and particularly of the jet observed for GW170817 [112,114]. In [115] it is shown that jets only transfer a small amount of energy to the ejecta. Further, we ignore the ejecta geometry and nonuniformity in velocity and opacity (see also $[106,116])$.

\section{GRAVITATIONAL WAVE EMISSION}

For all our simulations, we extract the GW signal at a fixed radius of $d \sim 1034 \mathrm{~km}$ using the Weyl formalism, with no extrapolation to infinity. In detail, we decompose the Weyl scalar $\Psi_{4}$ into spin-weighted spherical harmonics $\left|{ }_{-2} Y_{l m}(\theta, \phi)\right|$. Integrating twice in time yields the gravitational strain coefficients $h_{l m}=h_{l m}^{+}-i h_{l m}^{\times}$. The time integrations are performed using the method described in [74]. It effectively suppresses minor low-frequency offsets of numerical origin, which would otherwise get amplified in the integration.

GW strain and phase velocity for the three models are shown in Fig. 13. Only for model GM3 - 1.35, a BH was formed during the simulation, and one can see the corresponding decay of the amplitude around $14 \mathrm{~ms}$ after merger. From mass and spin of the $\mathrm{BH}$ produced for model $G M 3-1.35$, we obtain a ringdown frequency $f_{\mathrm{BH}}=6.52 \mathrm{kHz}$.

Comparing models $B L-1.35$ and $G M 3-1.35$, which have identical NS masses, we find a moderate influence of the EOS. The most prominent difference is the length of the postmerger GW signal, given by the remnant lifetime. For model $B L-1.35$, the remnant survives at least $5 \mathrm{~ms}$ longer (the corresponding GW signal is not fully visible in the figure since it needs time to reach the extraction radius). As discussed in Sec. IV, the collapse is probably delayed even longer.

The peak amplitude at merger is slightly larger for model $B L-1.35$. Subsequently, the amplitude decays faster for this model compared to model GM3 - 1.35. Another difference is that the frequency modulation, visible during the first few ms after merger, is stronger for model $B L-1.35$. Finally, the postmerger frequency is slightly higher for model $B L-1.35$, except close to the $\mathrm{BH}$ formation for model GM3 - 1.35.

Both the larger amplitude and stronger frequency modulation for model $B L-1.35$ might be related to the fact that the initial NSs are more compact (see Table II), such that the stars come into contact later and at higher velocities.

Comparing the two models with GM3 EOS, we find that the small mass difference has a significant impact on the length of the signal: the lighter model GM3 - 1.31 survives
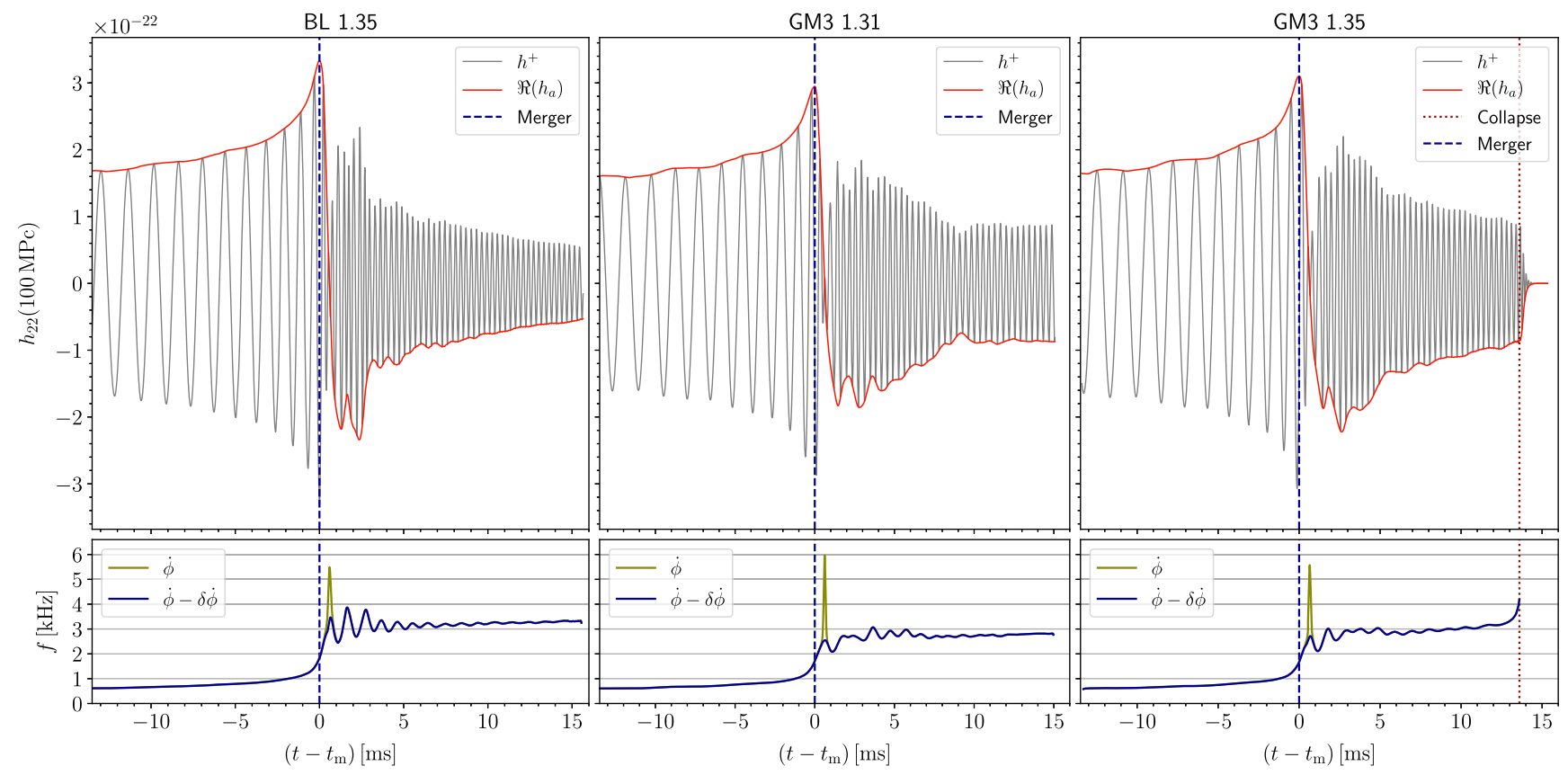

FIG. 13. GW strain (top panel) and instantaneous frequency (bottom panel) for our simulations. We only show the main contribution to the strain, given by the $l=m=2$ mode. The signal is scaled to a nominal source distance of $100 \mathrm{Mpc}$. The gray curve is the GW strain coefficient $h_{22}^{+}$. The red curve is the generalized amplitude accounting for over-modulation (see text). The yellow curve shows the instantaneous frequency computed from the phase of the strain, while the blue line shows the frequency without the contribution of the phase jump. The vertical dashed lines mark the merger times. The dotted vertical lines in the right panels mark the formation of an apparent horizon. 
at least $5 \mathrm{~ms}$ longer, probably more (see Sec. IV). The merger and postmerger amplitude is slightly larger for model GM3 - 1.35, and also the postmerger frequency is slightly higher for the latter. Both models show only weak modulation of the instantaneous frequency after merger. Model GM3 - 1.35 exhibits a faster drift towards higher frequencies, which is probably related to the shorter lifetime.

We also employ a scheme to detect phase jumps caused by over-modulation. The latter describes a signal $A(t) e^{i \phi(t)}$, with a slowly changing amplitude $A \in \mathbb{R}$ that is allowed to cross zero. For the case of GW signals, this could happen if the dominant multipole moment of the remnant in a corotating frame changes sign. Further details of the method can be found in [74].

As in $[73,74]$, we find that the strain minima occurring in our simulations directly after merger are likely caused by over-modulation. The generalized amplitude (obtained by allowing negative values, but no sudden phase jump, see [74]) is shown in the upper panels of Fig. 13. The lower panels shows that the sharp peaks in the phase velocity after merger (yellow curve) vanish when removing the contribution from over-modulation (blue curve).

The GW power spectra for the three models are shown in Fig. 14. For each, the dominant postmerger peak is clearly visible. Their frequencies are given in Table III and correspond to the instantaneous frequencies after merger discussed above.

To assess the detectability of the postmerger signal with the current LIGO detectors, we compare to [7]. The latter study compared several existing numerical relativity waveforms (which included the postmerger phase) to the instrument sensitivity during the detection of event GW170817. Assuming the same sky location and distance inferred for GW170817, the postmerger signal amplitudes were around

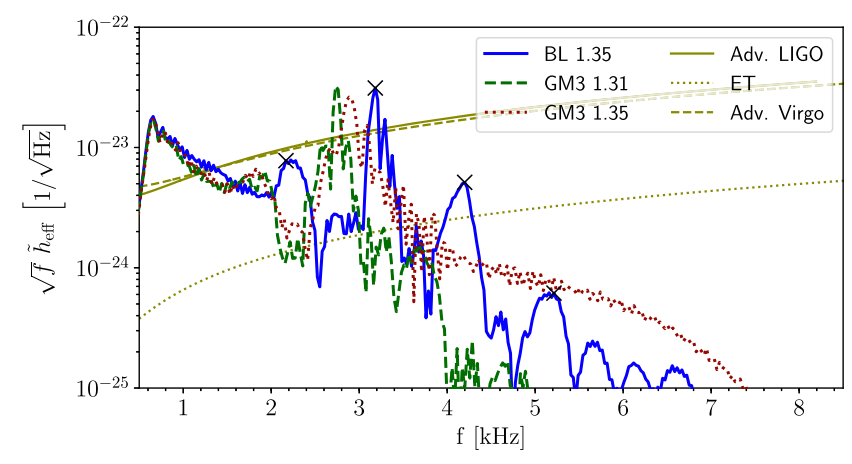

FIG. 14. Power spectrum of the GW strain for our simulations plotted together with the sensitivity curves for advanced LIGO and Virgo and the planned Einstein Telescope. The source is assumed at a distance $100 \mathrm{Mpc}$ from the detector, oriented with orbital angular momentum along the line of sight. The symbols mark the frequencies of the main peak and combination frequencies $f_{\mathrm{pm}}+n f_{\rho}$, where $n=-1,1,2$ and $f_{\rho}=1.0 \mathrm{kHz}$ is the quasiradial oscillation frequency. one order of magnitude too low for detection (see [7] for details).

The relevant quantities defined in [7] are a mean amplitude $h_{r s s}$ and mean frequency $\bar{f}$ in the frequency range $1-4 \mathrm{kHz}$. Note this range also includes part of the merger signal. For our model $B L-1.35$, we find $h_{r s s}=$ $0.26 \times 10^{-22} / \sqrt{\mathrm{Hz}}$ and $\bar{f}=2.36 \mathrm{kHz}$. Those are typical values compared to the cases studied in [7] (note, however, that we slightly underestimate the postmerger peak amplitude, since the $B L-1.35$ simulation ends when the GW amplitude is still large).

For comparison, Fig. 14 also shows the sensitivity curves of future detectors. Those indicate that the postmerger signals for our models at $100 \mathrm{Mpc}$ might be marginally detectable with advanced LIGO at design sensitivity, and should be detectable with the Einstein Telescope.

Besides the dominant postmerger peak, only model $B L-1.35$ exhibits significant secondary peaks. Despite the low amplitude, those peaks might be detectable with the future Einstein Telescope.

In principle, secondary peaks can have different interpretations. First, they could directly correspond to different oscillation modes excited in the remnant. Second, quasiradial oscillation can modulate the amplitude of the main $m=2$ mode (note that in a hypermassive NS, the radial oscillation frequency is typically lower than the $m=2$ frequency). The amplitude modulation then causes combination frequencies in the power spectrum, which differ from the frequency of the dominant $m=2$ mode by integer multiples of the quasiradial oscillation frequency [117]. Finally, changes in the remnant structure can affect the frequency of the dominant $m=2$ mode as well, causing broadening or splitting of the main peak (see Fig. 20 in [84] for an example).

For the case of model $B L-1.35$, the secondary peaks are caused by amplitude modulation. This can be seen in Fig. 14, marking the main frequency $f_{\mathrm{pm}}$ as well as combination frequencies with the radial oscillation frequency $f_{\rho}=1.0 \mathrm{kHz}$ (obtained from the power spectrum of the maximum density evolution). In [84], we noted that low frequency postmerger peaks can change strongly when removing the phase-jump during merger, due to cancellation effects with the late inspiral in the same frequency range. This is also the case here; however, we verified that the low-frequency peak is still present in the part of the signal starting at the phase-jump.

\section{CONCLUSIONS}

We presented simulations of binary neutron star mergers with the recently introduced BL EOS, which is derived from chiral effective field theory. For comparison, we carried out another simulation employing the GM3 EOS. Both evolve a system with equal mass NSs of $1.35 M_{\odot}$ each. To assess the influence of the mass, we study a third 
model with GM3 EOS and slightly lower NS masses of $1.31 M_{\odot}$.

Neither EOS includes thermal effects, while they both assume beta-equilibrium. We approximated the temperature dependence using a simple gamma law and ignore composition effects (a finite temperature version of the BL EOS will be the subject of future work). We also caution that our simulations do not include magnetic fields, and effective viscosity caused by magnetic fields or turbulence. This could influence the lifetime of the HMNS and the disk mass.

All models result in a hypermassive remnant, although only the heavier GM3 model formed a BH within the duration of our simulations, around $14 \mathrm{~ms}$ after merger. Not surprisingly, the mass had a strong impact on the lifetime. The lighter models survive at least $5 \mathrm{~ms}$ longer. The collapsing model supports a recent conjecture that the remnant mass distribution resembles a nonrotating NS in the core, and that HMNSs collapse once the corresponding TOV star reaches the maximum mass [73].

Both mass and EOS had a strong impact on the ejected mass. The BL EOS model ejects $\approx 6$ times more mass than the GM3 model with the same gravitational mass and $\approx 4$ times more than the lighter GM3 model. The reason might be the larger compactness of the initial NS with BL EOS, resulting in a later merger at larger velocities. In any case, we find that the BL model shows stronger oscillations after merger, and that most of the matter is ejected in several waves after the merger.

For all models, $90 \%$ of the mass is ejected within $50^{\circ}$ around the orbital plane. Due to our simplified treatment of the matter, we cannot estimate the composition of the ejecta, which have a large influence on the corresponding kilonova light curves. Using a standard range of opacities and order of magnitude analytical estimates based on ejecta mass and velocity, we find however that our models likely produce very different light curves. Accurate kilonova models would allow to distinguish the two EOSs from observations of the EM counterparts, provided that the masses are known. Comparing to kilonova models [107] describing the recent event GW170817, we find that the dynamical ejecta from our models are likely too slow and not massive enough to produce a similar contribution to the luminosity and spectral evolution.

The difference between the two EOSs is also evident in the postmerger $\mathrm{GW}$ signal. The main postmerger frequency for the $1.35 M_{\odot}$ systems is around $10 \%$ higher for the BL EOS than for the GM3 EOS. However, the mass also has a strong impact. The lighter GM3 model (with $\approx 3 \%$ lower total baryon mass) results in 5\% lower frequency. Detecting the postmerger signal would, therefore, clearly distinguish between the two EOSs considered here, provided the inspiral signal allows an accurate measurement of the total mass. At a fiducial distance of $100 \mathrm{Mpc}$, the postmerger peaks will be barely detectable with advanced LIGO at design sensitivity, but well resolved by the planned Einstein Telescope.

Due to the residual eccentricity and low number of orbits, we did not measure the tidal effects in our simulations, but we did compute the tidal deformabilities of our BL and GM3 NS models. Based on those, we expect that even the current LIGO detectors could likely distinguish between BL and GM3 EOS from the inspiral GW signal alone (compare [2]).

Last but not least, we studied the masses of the debris disks surrounding the final $\mathrm{BH}$. We compared two different definitions of disk mass. One is based on a cutoff density before $\mathrm{BH}$ formation, using the same value as in [75], and the other on a cutoff radius. We calibrated the cutoff radius to the mass remaining outside the $\mathrm{BH}$ for the collapsing model, and found that the density-based definition overestimates the final disk mass by around $60 \%$ for this model. Further, we found that the disk is not accreting on the remnant during the lifetime of the HMNSs, but instead increases due to mass shed by the remnant. Those ambiguities of disk mass could become relevant when estimating the potential contribution of disk winds to the kilonova signal, although the maximum fraction of ejected disk mass adds a larger uncertainty. Initial data used in the simulations presented in this paper and the gravitational wave signals can be downloaded from the supplemental material [[118]].

\section{ACKNOWLEDGMENTS}

We acknowledge PRACE for awarding us access to Marconi at CINECA, Italy (Grant No. 2016153613). Numerical calculations have been made also possible through a CINECA-INFN agreement, providing access to resources on MARCONI at CINECA. We also acknowledge the CINECA award under the ISCRA initiative, for the availability of high-performance computing resources and support (Grant No. IscrC_BNSTHM1).

\section{APPENDIX: TEMPERATURE EVOLUTION}

For the simulations in this work, we added thermal contributions to the cold BL and GM3 EOS by prescribing a simple gamma law of the form

$$
P(\rho, \epsilon)=P_{\text {cold }}(\rho)+\left(\Gamma_{\text {th }}-1\right)\left(\epsilon-\epsilon_{\text {cold }}\right) \rho,
$$

where $P_{\text {cold }}$ and $\epsilon_{\text {cold }}$ are given by the cold EOS tables (as functions of $\rho$ ), $\epsilon$ is the specific internal energy evolved by our code, and $\Gamma_{\text {th }}=1.8$.

In order to study the thermal evolution in our simulations, we extract the specific thermal energy $\epsilon_{\mathrm{th}}=\epsilon-$ $\epsilon_{\text {cold }}(\rho)$. In analogy to the ideal mono-atomic gas, we define a temperature 


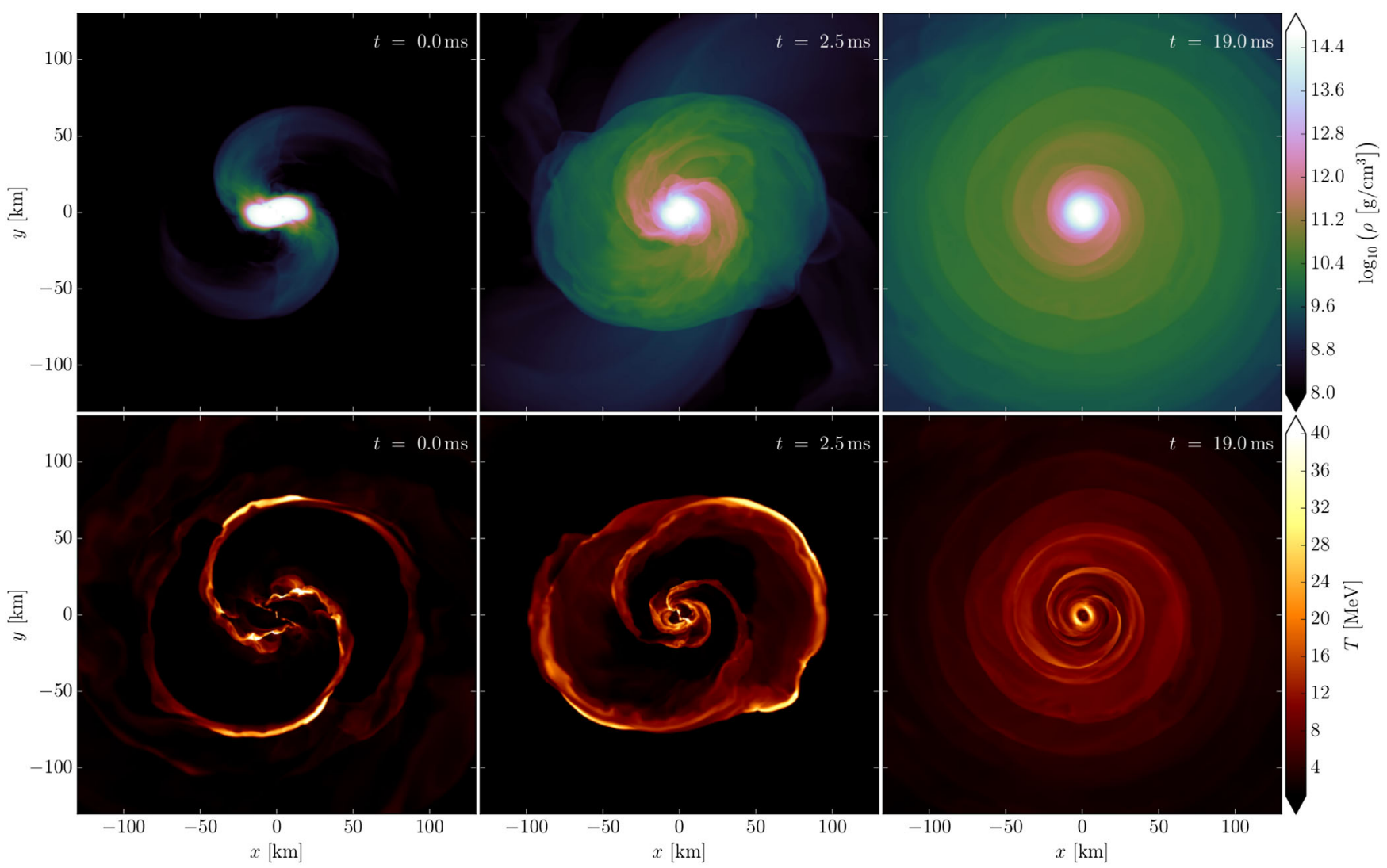

FIG. 15. Snapshots of the rest-mass density (top) and temperature (bottom) in the equatorial plane, for model BL-1.35 at merger time (left), $2.5 \mathrm{~ms}$ after merger (center), and $19 \mathrm{~ms}$ after merger (right).

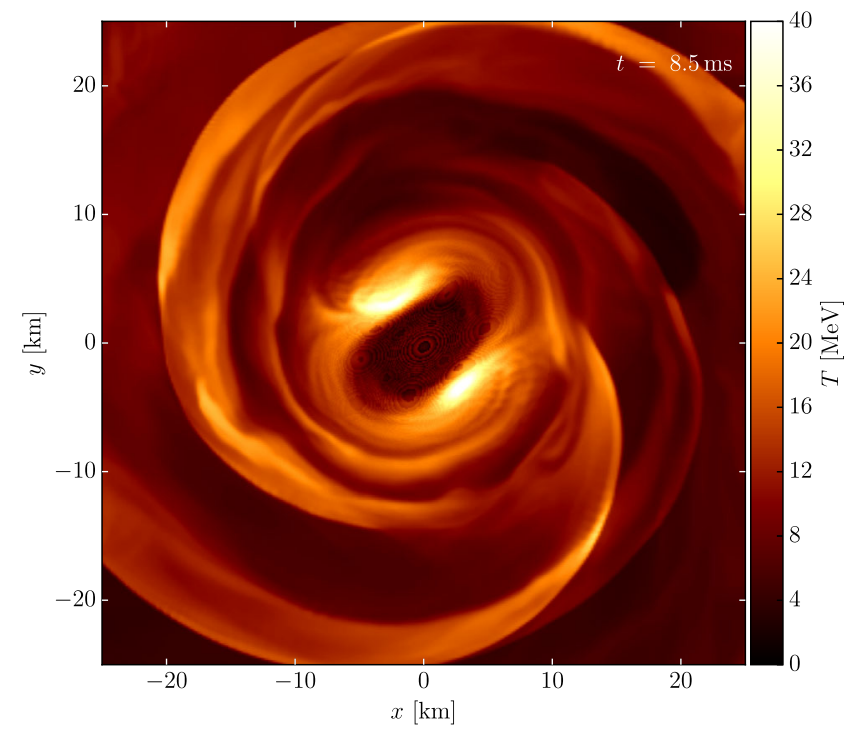

FIG. 16. Temperature inside the HMNS on the equatorial plane, $8.5 \mathrm{~ms}$ after merger, for model BL-1.35.

$$
T=\frac{2}{3} \frac{m_{b}}{k_{b}} \epsilon_{\mathrm{th}},
$$

where $k_{b}$ is the Boltzmann constant and $m_{b}$ the rest-mass of a nucleon.

In Fig. 15, we show the evolution of rest-mass density $\rho$ and temperature $T$ defined above on the equatorial plane during and after merger, for model $B L-1.35$. One can clearly see spiral shocks which heat the disk and the dynamical ejecta. The heating of the disk continues during the lifetime of the HMNS, a result we also observed in [74] for a different model. In previous works [74,84], employing a fully tabulated EOS including finite-temperature and composition effects, we observed a characteristic thermal pattern with two hot spots in the postmerger remnant. The models studied in this work exhibit a very similar structure. This is shown in Fig. 16, which can be compared to Fig. 12 in [74]. 
[1] B. P. Abbott et al. (LIGO Scientific Collaboration and Virgo Collaboration), Phys. Rev. Lett. 116, 061102 (2016).

[2] B. P. Abbott, R. Abbott, T. D. Abbott, F. Acernese, K. Ackley, C. Adams, T. Adams, P. Addesso, R. X. Adhikari, V. B. Adya et al., Phys. Rev. Lett. 119, 161101 (2017).

[3] B. P. Abbott, R. Abbott, T. D. Abbott, F. Acernese, K. Ackley, C. Adams, T. Adams, P. Addesso, R. X. Adhikari, V. B. Adya et al., Astrophys. J. Lett. 848, L12 (2017).

[4] D. A. Coulter et al., Science 358, 1556 (2017).

[5] B. P. Abbott, R. Abbott, T. D. Abbott, F. Acernese, K. Ackley, C. Adams, T. Adams, P. Addesso, R. X. Adhikari, V. B. Adya et al., Astrophys. J. Lett. 848, L13 (2017).

[6] B. D. Metzger, arXiv:1710.05931.

[7] B. P. Abbott, R. Abbott, T. D. Abbott, F. Acernese, K. Ackley, C. Adams, T. Adams, P. Addesso, R. X. Adhikari, V. B. Adya et al., Astrophys. J. Lett. 851, L16 (2017).

[8] B. D. Metzger and R. Fernández, Mon. Not. R. Astron. Soc. 441, 3444 (2014).

[9] N. R. Tanvir et al., Astrophys. J. Lett. 848, L27 (2017).

[10] B. Margalit and B. D. Metzger, Astrophys. J. Lett. 850, L19 (2017).

[11] A. Bauswein and H.-T. Janka, Phys. Rev. Lett. 108, 011101 (2012).

[12] D. Radice, S. Bernuzzi, W. Del Pozzo, L. F. Roberts, and C. D. Ott, Astrophys. J. Lett. 842, L10 (2017).

[13] N. Stergioulas, Living Rev. Relativity 6, 3 (2003).

[14] K. Hotokezaka, K. Kyutoku, H. Okawa, M. Shibata, and K. Kiuchi, Phys. Rev. D 83, 124008 (2011).

[15] B. Giacomazzo and R. Perna, Astrophys. J. Lett. 771, L26 (2013).

[16] A. L. Piro, B. Giacomazzo, and R. Perna, Astrophys. J. Lett. 844, L19 (2017).

[17] I. Bombaci and D. Logoteta, Astron. Astrophys. 609, A128 (2018).

[18] B. D. Day, Rev. Mod. Phys. 39 (1967).

[19] I. Bombaci, T. T. S. Kuo, and U. Lombardo, Phys. Rep. 242, 165 (1994).

[20] M. Baldo and G. F. Burgio, Rep. Prog. Phys. 75, 026301 (2012).

[21] N. K. Glendenning and S. A. Moszkowski, Phys. Rev. Lett. 67, 2414 (1991).

[22] Compact Stars: Nuclear Physics, Particle Physics, and General Relativity, edited by N. K. Glendenning (Springer, New York, 2000).

[23] N. K. Glendenning, Astrophys. J. 293, 470 (1985).

[24] I. Vidaña, D. Logoteta, C. Providência, A. Polls, and I. Bombaci, Europhys. Lett. 94, 11002 (2011).

[25] I. Bombaci and D. Logoteta, Mon. Not. R. Astron. Soc. 433, L79 (2013).

[26] I. Bombaci, D. Logoteta, I. Vidaña, and C. Providência, Europhys. J. A 52, 58 (2016).

[27] D. Logoteta, I. Bombaci, and A. Kievsky, Phys. Lett. B 758, 449 (2016).

[28] D. Logoteta, I. Bombaci, and A. Kievsky, Phys. Rev. C 94, 064001 (2016).

[29] A. Akmal, V. R. Pandharipande, and D. G. Ravenhall, Phys. Rev. C 58, 1804 (1998).

[30] M. Baldo, I. Bombaci, and G. F. Burgio, Astron. Astrophys. 328, 274 (1997).

[31] S. Weinberg, Physica A (Amsterdam) 96, 327 (1979).
[32] S. Weinberg, Phys. Lett. B 251, 288 (1990).

[33] S. Weinberg, Nucl. Phys. B363, 3 (1991).

[34] S. Weinberg, Phys. Lett. B 295, 114 (1992).

[35] E. Epelbaum, H.-W. Hammer, and U. G. Meißner, Rev. Mod. Phys. 81, 1773 (2009).

[36] R. Machleidt and D. R. Entem, Phys. Rep. 503, 1 (2011).

[37] J. W. Holt, N. Kaiser, and W. Weise, Prog. Part. Nucl. Phys. 73, 35 (2013).

[38] N. Kalantar-Nayestanaki, E. Epelbaum, J. G. Messchendorp, and A. Nogga, Rep. Prog. Phys. 75, 016301 (2012).

[39] H.-W. Hammer, N. A. Schwenk, and A. Schwenk, Rev. Mod. Phys. 85, 197 (2013).

[40] S. Binder et al. (Lenpic Collaboration), Phys. Rev. C 93, 044002 (2016).

[41] B. Friedman and V. R. Pandharipande, Nucl. Phys. A361, 502 (1981).

[42] W. Zuo, I. Bombaci, and U. Lombardo, Eur. Phys. J. A 50, 12 (2014).

[43] Z. H. Li and H.-J. Schulze, Phys. Rev. C 78, 028801 (2008).

[44] P. B. Demorest, T. Pennucci, S. M. Ransom, M. S. E. Roberts, and J.W. T. Hessels, Nature (London) 467, 1081 (2010).

[45] J. Antoniadis, P. C. C. Freire, N. Wex, T. M. Tauris, R. S. Lynch, M. H. van Kerkwijk, M. Kramer, C. Bassa, V. S. Dhillon, T. Driebe, J. W. T. Hessels, V. M. Kaspi, V. I. Kondratiev, N. Langer, T. R. Marsh, M. A. McLaughlin, T. T. Pennucci, S. M. Ransom, I. H. Stairs, J. van Leeuwen et al., Science 340, 1233232 (2013).

[46] P. Haensel and B. Pichon, Astron. Astrophys. 283, 313 (1994).

[47] F. Douchin and P. Haensel, Astron. Astrophys. 380, 151 (2001).

[48] Eur. Phys. J. A 50 (2014).

[49] M. Baldo and G. F. Burgio, Prog. Part. Nucl. Phys. 91, 203 (2016).

[50] I. Bombaci and U. Lombardo, Phys. Rev. C 44, 1892 (1991).

[51] J. M. Lattimer, Gen. Relativ. Gravit. 46, 1713 (2014).

[52] J. M. Lattimer and M. Prakash, Astrophys. J. 550, 426 (2001).

[53] J. M. Lattimer and Y. Lim, Astrophys. J. 771, 51 (2013).

[54] I. Tews, J. M. Lattimer, A. Ohnishi, and E. E. Kolomeitsev, Astrophys. J. 848, 105 (2017).

[55] K.-H. Bennemann and J. B. Ketterson, Novel Superfluids, (Oxford Scholarship Online, 2015), Vol. 2, Chap. 12.

[56] K. Kiuchi, K. Kyutoku, Y. Sekiguchi, M. Shibata, and T. Wada, Phys. Rev. D 90, 041502 (2014).

[57] A. Bauswein, H. Janka, and R. Oechslin, Phys. Rev. D 82, 084043 (2010).

[58] I. Bombaci, Astron. Astrophys. 305, 871 (1996).

[59] M. Prakash, I. Bombaci, M. Prakash, P. J. Ellis, J. M. Lattimer, and R. Knorren, Phys. Rep. 280, 1 (1997).

[60] A. Bauswein, T. W. Baumgarte, and H.-T. Janka, Phys. Rev. Lett. 111, 131101 (2013).

[61] T. Hinderer, B. D. Lackey, R. N. Lang, and J. S. Read, Phys. Rev. D 81, 123016 (2010).

[62] E. Gourgoulhon, P. Grandclement, K. Taniguchi, J.-A. Marck, and S. Bonazzola, Phys. Rev. D 63, 064029 (2001). 
[63] K. Taniguchi and E. Gourgoulhon, Phys. Rev. D 66, 104019 (2002).

[64] F. Galeazzi, W. Kastaun, L. Rezzolla, and J. A. Font, Phys. Rev. D 88, 064009 (2013).

[65] D. Alic, W. Kastaun, and L. Rezzolla, Phys. Rev. D 88, 064049 (2013).

[66] T. W. Baumgarte and S. L. Shapiro, Phys. Rev. D 59, 024007 (1998).

[67] M. Shibata and T. Nakamura, Phys. Rev. D 52, 5428 (1995).

[68] T. Nakamura, K. Oohara, and Y. Kojima, Prog. Theor. Phys. Suppl. 90, 1 (1987).

[69] F. Löffler, J. Faber, E. Bentivegna, T. Bode, P. Diener, R. Haas, I. Hinder, B. C. Mundim, C. D. Ott, E. Schnetter, G. Allen, M. Campanelli, and P. Laguna, Classical Quantum Gravity 29, 115001 (2012).

[70] E. Schnetter, S. H. Hawley, and I. Hawke, Classical Quantum Gravity 21, 1465 (2004).

[71] F. Banyuls, J. A. Font, J. M. Ibáñez, J. M. Martí, and J. A. Miralles, Astrophys. J. 476, 221 (1997).

[72] A. Harten, P. D. Lax, and B. van Leer, SIAM Rev. 25, 35 (1983).

[73] R. Ciolfi, W. Kastaun, B. Giacomazzo, A. Endrizzi, D. M. Siegel, and R. Perna, Phys. Rev. D 95, 063016 (2017).

[74] W. Kastaun, R. Ciolfi, and B. Giacomazzo, Phys. Rev. D 94, 044060 (2016).

[75] D. Radice, A. Perego, F. Zappa, and S. Bernuzzi, Astrophys. J. Lett. 852, L29 (2018).

[76] B. D. Metzger, E. Quataert, and T. A. Thompson, Mon. Not. R. Astron. Soc. 385, 1455 (2008).

[77] B. D. Metzger, A. L. Piro, and E. Quataert, Mon. Not. R. Astron. Soc. 396, 304 (2009).

[78] R. Fernández, D. Kasen, B. D. Metzger, and E. Quataert, Mon. Not. R. Astron. Soc. 446, 750 (2015).

[79] O. Just, M. Obergaulinger, and H.-T. Janka, Mon. Not. R. Astron. Soc. 453, 3386 (2015).

[80] D. M. Siegel and B. D. Metzger, Astrophys. J. 858, 52 (2018).

[81] R. Fernández and B. D. Metzger, Mon. Not. R. Astron. Soc. 435, 502 (2013).

[82] W. Kastaun and F. Galeazzi, Phys. Rev. D 91, 064027 (2015).

[83] A. Endrizzi, R. Ciolfi, B. Giacomazzo, W. Kastaun, and T. Kawamura, Classical Quantum Gravity 33, 164001 (2016).

[84] W. Kastaun, R. Ciolfi, A. Endrizzi, and B. Giacomazzo, Phys. Rev. D 96, 043019 (2017).

[85] M. Hanauske, K. Takami, L. Bovard, L. Rezzolla, J. A. Font, F. Galeazzi, and H. Stöcker, Phys. Rev. D 96, 043004 (2017).

[86] M. Shibata, K. Kiuchi, and Y.-i. Sekiguchi, Phys. Rev. D 95, 083005 (2017).

[87] K. Kiuchi, P. Cerdá-Durán, K. Kyutoku, Y. Sekiguchi, and M. Shibata, Phys. Rev. D 92, 124034 (2015).

[88] T. Kawamura, B. Giacomazzo, W. Kastaun, R. Ciolfi, A. Endrizzi, L. Baiotti, and R. Perna, Phys. Rev. D 94, 064012 (2016).

[89] M. Ruiz, R. N. Lang, V. Paschalidis, and S. L. Shapiro, Astrophys. J. 824, L6 (2016).
[90] R. Ciolfi, arXiv:1804.03684.

[91] A. Rowlinson, P. T. O’Brien, B. D. Metzger, N. R. Tanvir, and A. J. Levan, Mon. Not. R. Astron. Soc. 430, 1061 (2013).

[92] R. Ciolfi and D. M. Siegel, Astrophys. J. Lett. 798, L36 (2015).

[93] A. Murguia-Berthier, G. Montes, E. Ramirez-Ruiz, F. De Colle, and W. H. Lee, Astrophys. J. Lett. 788, L8 (2014).

[94] H. Nagakura, K. Hotokezaka, Y. Sekiguchi, M. Shibata, and K. Ioka, Astrophys. J. Lett. 784, L28 (2014).

[95] O. Just, M. Obergaulinger, H.-T. Janka, A. Bauswein, and N. Schwarz, Astrophys. J. Lett. 816, L30 (2016).

[96] A. Murguia-Berthier, E. Ramirez-Ruiz, G. Montes, F. De Colle, L. Rezzolla, S. Rosswog, K. Takami, A. Perego, and W. H. Lee, Astrophys. J. Lett. 835, L34 (2017).

[97] D. Martin, A. Perego, W. Kastaun, and A. Arcones, Classical Quantum Gravity 35, 034001 (2018).

[98] Y. Sekiguchi, K. Kiuchi, K. Kyutoku, and M. Shibata, Phys. Rev. D 91, 064059 (2015).

[99] K. Hotokezaka, K. Kiuchi, K. Kyutoku, T. Muranushi, Y.-I. Sekiguchi, M. Shibata, and K. Taniguchi, Phys. Rev. D 88, 044026 (2013).

[100] R. Oechslin, H.-T. Janka, and A. Marek, Astron. Astrophys. 467, 395 (2007).

[101] D. Grossman, O. Korobkin, S. Rosswog, and T. Piran, Mon. Not. R. Astron. Soc. 439, 757 (2014).

[102] L.-X. Li and B. Paczyński, Astrophys. J. Lett. 507, L59 (1998).

[103] B. D. Metzger, G. Martínez-Pinedo, S. Darbha, E. Quataert, A. Arcones, D. Kasen, R. Thomas, P. Nugent, I. V. Panov, and N. T. Zinner, Mon. Not. R. Astron. Soc. 406, 2650 (2010).

[104] L. F. Roberts, D. Kasen, W. H. Lee, and E. Ramirez-Ruiz, Astrophys. J. Lett. 736, L21 (2011).

[105] J. Barnes and D. Kasen, Astrophys. J. 775, 18 (2013).

[106] V. A. Villar, J. Guillochon, E. Berger, B. D. Metzger, P. S. Cowperthwaite, M. Nicholl, K. D. Alexander, P. K. Blanchard, R. Chornock, T. Eftekhari, W. Fong, R. Margutti, and P. K. G. Williams, Astrophys. J. Lett. 851, L21 (2017).

[107] D. Kasen, B. Metzger, J. Barnes, E. Quataert, and E. Ramirez-Ruiz, Nature (London) 551, 80 (2017).

[108] L. Dessart, C. D. Ott, A. Burrows, S. Rosswog, and E. Livne, Astrophys. J. 690, 1681 (2009).

[109] D. M. Siegel, R. Ciolfi, and L. Rezzolla, Astrophys. J. Lett. 785, L6 (2014).

[110] D. M. Siegel and B. D. Metzger, Phys. Rev. Lett. 119, 231102 (2017).

[111] K. P. Mooley, E. Nakar, K. Hotokezaka, G. Hallinan, A. Corsi, D. A. Frail, A. Horesh, T. Murphy, E. Lenc, D. L. Kaplan, K. De, D. Dobie, P. Chandra, A. Deller, O. Gottlieb, M. M. Kasliwal, S. R. Kulkarni, S. T. Myers, S. Nissanke, T. Piran et al., Nature (London) 554, 207 (2018)

[112] D. Lazzati, R. Perna, B. J. Morsony, D. Lopez-Camara, M. Cantiello, R. Ciolfi, B. Giacomazzo, and J. C. Workman, Phys. Rev. Lett. 120, 241103 (2018).

[113] E. Berger, Annu. Rev. Astron. Astrophys. 52, 43 (2014). 
[114] R. Margutti, K. D. Alexander, X. Xie, L. Sironi, B. D. Metzger, A. Kathirgamaraju, W. Fong, P. K. Blanchard, E. Berger, A. MacFadyen, D. Giannios, C. Guidorzi, A. Hajela, R. Chornock, P. S. Cowperthwaite, T. Eftekhari, M. Nicholl, V. A. Villar, P. K. G. Williams, and J. Zrake, Astrophys. J. Lett. 856, L18 (2018).

[115] P. C. Duffell, E. Quataert, D. Kasen, and H. Klion, arXiv: 1806.10616.
[116] A. Perego, D. Radice, and S. Bernuzzi, Astrophys. J. Lett. 850, L37 (2017).

[117] N. Stergioulas, A. Bauswein, K. Zagkouris, and H.-T. Janka, Mon. Not. R. Astron. Soc. 418, 427 (2011).

[118] See Supplemental Material at http://link.aps.org/ supplemental/10.1103/PhysRevD.98.043015 for initial data and gravitational wave signals. 